Old Dominion University

ODU Digital Commons

2-2019

Correction: New Approaches for Assessing Squid Fin Motions:

Coupling Proper Orthogonal Decomposition With Volumetric

Particle Tracking Velocimetry (doi:10.1242/jeb.176750)

lan K. Bartol

Paul S. Krueger

Carly A. York

Joseph T. Thompson

Follow this and additional works at: https://digitalcommons.odu.edu/biology_fac_pubs

Part of the Biomechanics Commons, and the Marine Biology Commons 


\section{Correction: New approaches for assessing squid fin motions: coupling proper orthogonal decomposition with volumetric particle tracking velocimetry (doi:10.1242/jeb.176750)}

Ian K. Bartol, Paul S. Krueger, Carly A. York and Joseph T. Thompson

There was an error published in J. Exp. Biol. (2018) 221, jeb176750 (doi:10.1242/jeb.176750).

In Figs 6-10, the vorticity magnitude colour bar was incorrect. The original bar (left) and corrected bar (right) appear below.
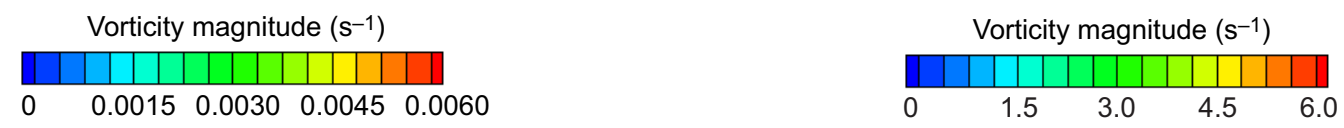

Figs 6-8 and 10
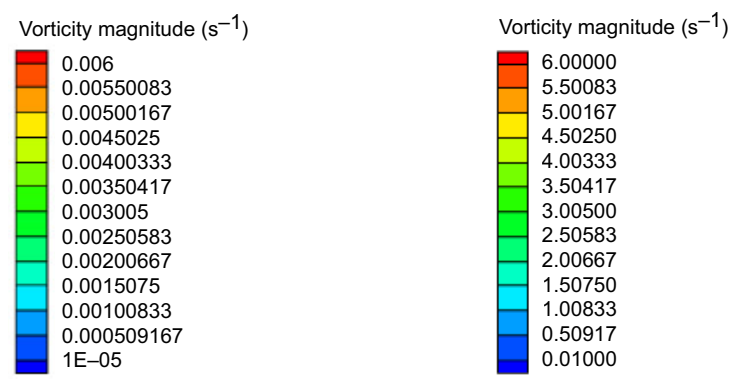

Fig. 9

Both the online full-text and PDF versions have been updated.

The authors apologise for these errors and any inconvenience they may have caused. 


\title{
New approaches for assessing squid fin motions: coupling proper orthogonal decomposition with volumetric particle tracking velocimetry
}

\author{
Ian K. Bartol ${ }^{1, *}$, Paul S. Krueger ${ }^{2}$, Carly A. York ${ }^{3}$ and Joseph T. Thompson ${ }^{4}$
}

\begin{abstract}
Squid, which swim using a coupled fin/jet system powered by muscular hydrostats, pose unique challenges for the study of locomotion. The high flexibility of the fins and complex flow fields generated by distinct propulsion systems require innovative techniques for locomotive assessment. For this study, we used proper orthogonal decomposition (POD) to decouple components of the fin motions and defocusing digital particle tracking velocimetry (DDPTV) to quantify the resultant 3D flow fields. Kinematic footage and DDPTV data were collected from brief squid, Lolliguncula brevis [3.1-6.5 cm dorsal mantle length (DML)], swimming freely in a water tunnel at speeds of 0.39-7.20 DML s ${ }^{-1}$. Both flap and wave components were present in all fin motions, but the relative importance of the wave components was higher for arms-first swimming than for tail-first swimming and for slower versus higher speed swimming. When prominent wave components were present, more complex interconnected vortex ring wakes were observed, while fin movements dominated by flapping resulted in more spatially separated vortex ring patterns. Although the jet often produced the majority of the thrust for steady rectilinear swimming, our results demonstrated that the fins can contribute more thrust than the jet at times, consistently produce comparable levels of lift to the jet during arms-first swimming, and can boost overall propulsive efficiency. By producing significant drag signatures, the fins can also aid in stabilization and maneuvering. Clearly, fins play multiple roles in squid locomotion, and when coupled with the jet, allow squid to perform a range of swimming behaviors integral to their ecological success.
\end{abstract}

KEY WORDS: Cephalopod, Vorticity, Fins, Flapping, Undulation, Proper orthogonal decomposition, 3D velocimetry

\section{INTRODUCTION}

Squid swim, unlike most other nektonic animals, using a combination of coordinated pulsed jetting and fin movements (Fig. 1). This hybrid system represents an intriguing contrast from swimmers that rely on appendages and/or body motions for propulsion because it includes both oscillatory/undulatory propulsors and a pulsed jet that can be vectored in any direction in a hemisphere beneath the body. The unique combination of jetting and finning allows squid to switch

${ }^{1}$ Department of Biological Sciences, Old Dominion University, Norfolk, VA 23529, USA. ${ }^{2}$ Department of Mechanical Engineering, Southern Methodist University, Dallas, TX 75275, USA. ${ }^{3}$ Department of Biology, Lenoir-Rhyne University, Hickory, NC 28601, USA. ${ }^{4}$ Department of Biology, Franklin and Marshall College, Lancaster. PA 17603, USA.

*Author for correspondence (ibartol@odu.edu)

(D) I.K.B., 0000-0001-9006-8727

Received 27 December 2017; Accepted 14 May 2018 rapidly from swimming forward (arms-first) to backward (tail-first), hold station in turbulent flows, ascend vertically and navigate structurally complex habitats (Bartol et al., 2001a,b; Hanlon et al., 1983; Vecchione and Roper, 1991). Squid are highly maneuverable, having the smallest length-specific turning radii of any aquatic taxa measured to date (Jastrebsky et al., 2016), and can track and capture prey with high efficiency (>80\%; Jastrebsky et al., 2017) while avoiding predators when their sensory and locomotive systems are intact (York and Bartol, 2014, 2016; York et al., 2016).

The hydrodynamics of the pulsed jet have been studied in several species of neritic and oceanic squids (Anderson and DeMont, 2000; Anderson and Grosenbaugh, 2005; Bartol et al., 2001b, 2008, 2009a,b, 2016; Johnson et al., 1972; O’Dor, 1988), but much less is known about the hydrodynamics of the other propulsive component in this dual-mode system, namely the fins (but see Bartol et al., 2016; Stewart et al., 2010). Fin morphology and function vary widely among squids. Some squids have short rhomboidal fins (e.g. Illex illecebrosus), while others have pronounced lateral fins that extend along the entire mantle margin (e.g. Sepioteuthis lessoniana, Thysanoteuthis rhombus) (O'Dor et al., 1995; Hoar et al., 1994). In some fast-swimming oceanic squids, the fins serve primarily as stabilizers and rudders, whereas in some inshore squids, the fins are used for not only stability and steering but also propulsion (Anderson and DeMont, 2005; Bartol et al., 2001b; Hoar et al., 1994; O’Dor, 1988). Many deep-sea squids appear to rely heavily on their fins for locomotion, with the jet playing a more subordinate role (Vecchione et al., 2001, 2002). Some squids, such as Sthenoteuthis pteropus, Sthenoteuthis oualaniensis and Ommastrephes bartramii, even use their fins as wings for aerial gliding and possibly flight (Muramatsu et al., 2013; O’Dor et al., 2013).

All squid fins are supported by a muscular hydrostatic skeleton (Kier, 1989; Kier and Smith, 1985; Kier and Thompson, 2003), which is a $3 \mathrm{D}$, tightly packed array of muscle and connective tissue that serves as the support for and the effector of movement. Although lacking the rigid skeletal elements of fish fins and, therefore, the ability to use levers to adjust force and actuation speed, the muscular hydrostatic fins nevertheless are capable of highly diverse movements with high degrees of freedom. Movements ranging from simple flapping to multi-wave, bi-directional undulations have been observed in squid (Anderson and DeMont, 2005; Bartol et al., 2001b; Hoar et al., 1994; Vecchione et al., 2001, 2002). This range of motion provides propulsive and control benefits that complement the vectored jet system, as demonstrated in cruising, maneuverability and predatorattack studies (Bartol et al., 2016, Jastrebsky et al., 2016, 2017).

The complex fluid-structure interactions associated with these muscular hydrostatic systems pose unique challenges for researchers interested in studying the kinematics and hydrodynamics of fin motions in squid. Similar challenges emerge when investigating fluid-structure interactions in other complex highly deformable 


\begin{tabular}{|c|c|}
\hline \multicolumn{2}{|c|}{ List of symbols and abbreviations } \\
\hline DDPTV & defocusing digital particle tracking velocimetry \\
\hline DML & dorsal mantle length $(\mathrm{cm})$ \\
\hline E & total kinetic energy $\left(\mathrm{kg} \mathrm{m}^{2} \mathrm{~s}^{-2}\right)$ \\
\hline$E_{\mathrm{j}}$ & rate of excess kinetic energy shed by the jet $\left(\mathrm{kg} \mathrm{m}^{2} \mathrm{~s}^{-3}\right)$ \\
\hline$E_{f}$ & rate of excess kinetic energy shed by the fin $\left(\mathrm{kg} \mathrm{m}^{2} \mathrm{~s}^{-3}\right)$ \\
\hline $\bar{F}_{\mathrm{fl}}$ & time-averaged fin lift $(\mathrm{N})$ \\
\hline $\bar{F}_{\mathrm{ft}}$ & time-averaged fin thrust $(\mathrm{N})$ \\
\hline $\bar{F}_{\mathrm{jl}}$ & time-averaged jet lift $(\mathrm{N})$ \\
\hline$F_{\mathrm{jt}}$ & time-averaged jet thrust $(\mathrm{N})$ \\
\hline I & impulse ( $\mathrm{N} \mathrm{s})$ \\
\hline $\mathbf{n}$ & $\begin{array}{l}\text { unit vector normal to and oriented outward from a closed } \\
\text { surface }\end{array}$ \\
\hline POD & proper orthogonal decomposition \\
\hline $\operatorname{Re}$ & Reynolds number \\
\hline SV & mode singular values \\
\hline SVD & singular value decomposition \\
\hline$q_{k}(t)$ & temporal variation of spatial shape $(\mathrm{cm})$ \\
\hline$S$ & surface bounding volume $V\left(\mathrm{~cm}^{2}\right)$ \\
\hline$T$ & period of fin or jet cycle (s) \\
\hline$t$ & time (s) \\
\hline$U$ & swimming velocity $\left(\mathrm{cm} \mathrm{s}^{-1}\right.$ or $\left.\mathrm{m} \mathrm{s}^{-1}\right)$ \\
\hline $\mathbf{u}$ & velocity vector $\left(\mathrm{m} \mathrm{s}^{-1}\right)$ \\
\hline$|u|$ & velocity magnitude $\left(\mathrm{m} \mathrm{s}^{-1}\right)$ \\
\hline V & volume bounding a vortex $\left(\mathrm{cm}^{3}\right)$ \\
\hline $\mathbf{v}_{k}(\mathbf{x})$ & spatial shape (unitless) \\
\hline $\mathbf{x}$ & position vector ( $\mathrm{cm}$ or $\mathrm{m})$ \\
\hline$\hat{\mathbf{x}}$ & unit vector opposite the direction of tunnel flow (unitless) \\
\hline$\hat{\mathbf{y}}$ & unit vector in the vertical direction (unitless) \\
\hline $\mathbf{z}(\mathbf{x}, t)$ & fin motion $(\mathrm{cm})$ \\
\hline$\sigma_{k}$ & singular value $(\mathrm{cm})$ \\
\hline$\eta$ & propulsive efficiency (\%) \\
\hline$\omega$ & vorticity vector $\left(\mathrm{s}^{-1}\right)$ \\
\hline$\rho$ & fluid density $\left(\mathrm{kg} \mathrm{m}^{-3}\right)$ \\
\hline
\end{tabular}

surfaces, such as fish fins and insect, bird and bat wings (Riskin et al., 2008; Lentink and Dickinson, 2009; Tangorra et al., 2010; Neveln et al., 2014; Crandell and Tobalske, 2015; Di Santo et al., 2017). To address this complexity, tools to identify spatial and temporal components of fin motions and to quantify $3 \mathrm{D}$ flows shed from the fins are needed.

We investigated the following questions: (1) are flap and wave-like features identifiable in active fins?; (2) are there differences in fin behavior with swimming orientation and speed?; and (3) do different fin motions produce different wake structures and forces? To answer these questions, we employed two new approaches in tandem that allowed us to comprehensively assess cephalopod fin kinematics and hydrodynamics. First, proper orthogonal decomposition (POD) was used to identify the major spatial and temporal modes of fin motions. Second, defocusing digital particle tracking velocimetry (DDPTV), a volumetric velocimetry technique, was used to quantify flow features around the fins. For these investigations, we focused on the brief squid, Lolliguncula brevis, because it has rounded, highly active fins that are capable of producing a wide suite of motions.

\section{MATERIALS AND METHODS \\ Animals}

Brief squid, Lolliguncula brevis (Blainville 1823), were captured by trawl at the Virginia Institute of Marine Science Eastern Shore Laboratory in Wachapreague, VA, USA. The squid were transported to Old Dominion University's Marine Aquatics Facility and maintained in seawater systems using protocols described in previous studies (Bartol et al., 2009b, 2016). For this study, $43 \mathrm{~L}$. brevis ranging in size from 3.1 to $6.5 \mathrm{~cm}$ dorsal mantle length
(DML) (4.4-26.6 g) were considered. Squid were fed a diet of live Palaemonetes pugio and Fundulus heteroclitus and were maintained for at least $72 \mathrm{~h}$ prior to experimentation.

\section{Experiments}

High-speed video and DDPTV data were collected simultaneously using protocols similar to those described in Bartol et al. (2016). For convenience, we describe some general aspects of the approach here. Experiments were conducted in a water tunnel with a $15 \times 15 \times 44 \mathrm{~cm}$ working section [Model 502(s), Engineering Laboratory Design, Lake City, MN, USA] filled with seawater (30 ppt) containing suspended reflective seeding particles (polyamide, $50 \mu \mathrm{m}$, Dantec Dynamics, Skovlunde, Denmark). Each squid was placed in the tunnel separately and exposed to flow speeds ranging from 3 to $25 \mathrm{~cm} \mathrm{~s}^{-1}$, with trials terminating when the squid could no longer keep pace with free-stream flow. Images of the squid body and fins were collected using three Falcon high-speed cameras (Teledyne Dalsa, Inc., Waterloo, ON, Canada; $1400 \times 1200$ pixels, 100 frames s$^{-1}$ ) while images of flow around the swimming squid were collected simultaneously using a V3V-8000 probe (TSI, Inc., Shoreview, MN, USA). Although high-speed images from multiple perspectives were collected, only lateral footage of fin motions was analyzed in this study, with the other perspectives being used to ensure that the squid was near the middle of the working section and away from tunnel walls in selected sequences.

Triggering of the high-speed cameras was achieved using an onboard counter from a CLSAS capture card and Streams 5 software (IO Industries, London, ON, Canada), which were also used to save kinematic images directly to an array of internal hard drives. The highspeed cameras were illuminated using halogen lights with filters for transmitting light at red $(>600 \mathrm{~nm})$ wavelengths. To prevent overexposure of frames from the pulsed laser, we used a notch filter with each Falcon camera to block the laser's $532 \mathrm{~nm}$ output wavelength. INSIGHT 4G V3V software (TSI, Inc.) was used to collect paired DDPTV images of flows around the squid (time separation between paired images, $\Delta t=2.0 \mathrm{~ms}$ ) at $7 \mathrm{~Hz}$, with probe timing signals being produced by a TSI synchronizer. The images were illuminated with two pulsed Nd:YAG lasers (LABest, Optronics, Beijing, China) triggered using a BNC-565 pulse generator (Berkeley Nucleonics, San Rafael, CA, USA). The V3V-8000 probe was outfitted with optical filters so that only laser light illumination at 532 $\pm 5 \mathrm{~nm}$ reached the DDPTV CCD sensors. Synchronization of laser firing, V3V-8000 probe image collection and high-speed image collection were achieved using a master TTL signal produced by a PCI NI-6602 timing board, National Instruments timing software and keyboard triggers (National Instruments, Austin, TX, USA).

\section{POD processing}

POD, which has been used for modeling, analyzing and reconstructing complex motions in sunfish pectoral fins and bat wings (Bozkurttas et al., 2006, 2009; Riskin et al., 2008), was used to decompose and identify dominant fin motions in the swimming squid. For this study, the singular value decomposition (SVD) method was used for POD analysis. POD deconstructs the fin motion, $\mathbf{z}(\mathbf{x}, t)$, into spatial $N$ shapes, $\mathbf{v}_{k}(\mathbf{x}) \quad(k=1,2, \ldots, N)$, and corresponding temporal variation of those shapes, $q_{k}(t)$, and ranks them according to their impact on the total measured motion. The ranking is done according to the singular values, $\sigma_{k}$, of the modes, which are related to $q_{k}(t)$ as:

$$
\sigma_{k}^{2}=\int q_{k}^{2}(t) \mathrm{d} t
$$


A

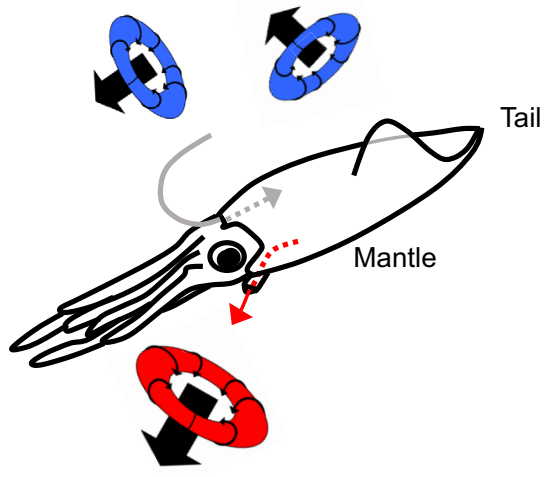

Tail-first swimming

Arms-first swimming
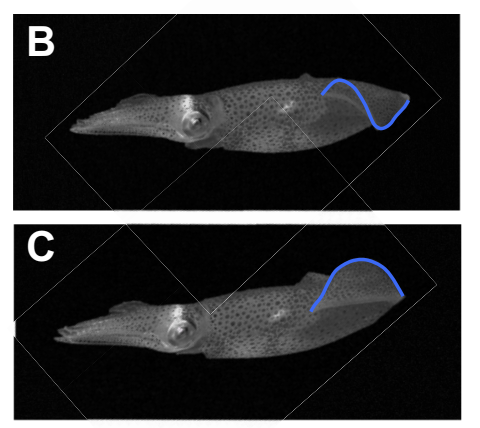

Flow
Fig. 1. Squid positioning and propulsive mechanisms during swimming. (A-C) Tail-first swimming and (D-F) arms-first swimming. To swim, squid use (1) a pulsed jet that involves the intake of water into the mantle (gray arrows) and subsequent expulsion of water through a vectored funnel (red arrows) and (2) fin motions that include undulatory $(\mathrm{B}, \mathrm{E})$ and flapping $(\mathrm{C}, \mathrm{F})$ components. Vortex rings are common structures observed in the wake of the fins (shown in blue) and jet (shown in red).
D

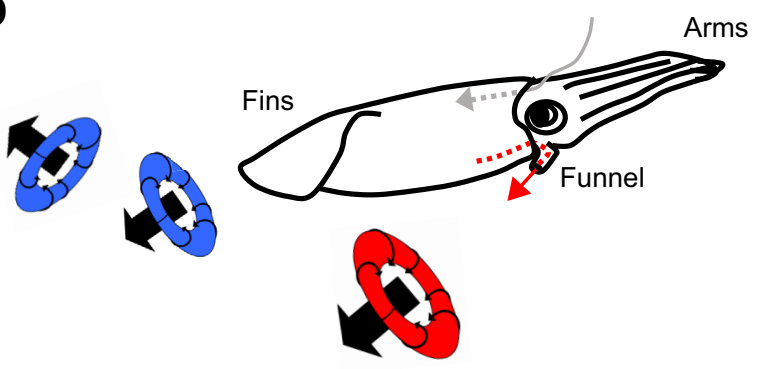

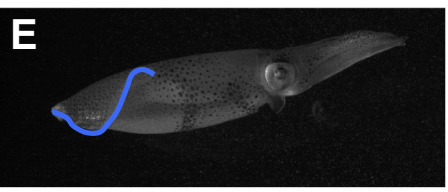

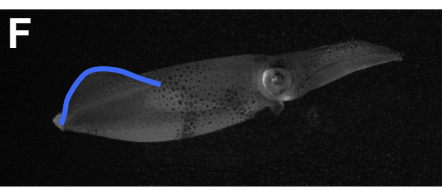

where the integration is taken over the duration of the motion. Physically, the square of the singular values is representative of the total kinetic energy associated with the motion of each mode.

Using custom-written in-house Matlab code, the lateral fin margin was traced beginning at the most downstream fin region and ending at the most upstream fin region for both swimming orientations. The code interpolated the data onto 25 evenly spaced points along the traced fin margin between the starting and ending point. These points, $\mathbf{z}(\mathbf{x}, t)$, were decomposed over orthogonal (i.e. independent) spatial modes:

$$
\mathbf{z}(\mathbf{x}, t)=\sum_{k=1}^{N} q_{k}(t) \mathbf{v}_{k}(\mathbf{x}) .
$$

The amplitude, phase and frequency information for each POD mode are contained in the $q_{k}(t)$ coefficients. The decomposition was performed using SVD in Matlab. POD data were smoothed by removing outliers (outlier window of 3 ) and applying a fourth-order Butterworth filter $(10 \mathrm{~Hz}$ cutoff). The highest fundamental frequency observed was $8.1 \mathrm{~Hz}$. The dominant frequencies and wavelengths for the POD modes were extracted from mode amplitude and mode shape data, respectively, by computing the pseudospectrum of the relevant signal using the MUSIC algorithm (Marple, 1987) as implemented in the Matlab function pmusic. Wavelengths were reported relative to squid fin length.

POD analysis was performed on 20 tail-first and 23 armsfirst swimming sequences, each with $\geq 3$ successive fin cycles (upstroke+downstroke). To preserve independence in statistical tests, different squid were considered in each sequence $(N=43)$. The data were divided into three speed ranges: $<1.75,1.75-2.75$ and $>2.75 \mathrm{DML} \mathrm{s}^{-1}$, with at least five sequences selected within each speed range. The criteria used for sequence selection were:
(1) the squid had to swim at least $2 \mathrm{~cm}$ away from the tunnel walls, (2) the squid had to swim parallel to oncoming flow and (3) the squid had to use both jet propulsion and consistent fin movements during swimming. We computed swimming velocity $\left(U ; \mathrm{cm} \mathrm{s}^{-1}\right)$ by measuring net displacement (positive or negative) along the $x$-axis per unit time over at least 3 fin cycles and adding this to the background water tunnel velocity. These corrections resulted in the expansion of the speed range considered $\left(2.14-46.2 \mathrm{~cm} \mathrm{~s}^{-1}\right)$.

\section{DDPTV processing}

A subset of the kinematic sequences considered for POD analysis was considered for DDPTV processing (17 of 23 arms-first sequences; 15 of 20 tail-first sequences). A subset was used because some kinematic sequences involved squid that were partially out of the DDPTV sampling volume or too close to the DDPTV video frame edge for reliable imaging of the complete fin and jet wake. Within each kinematic sequence, 3-16 successive DDPTV images were processed. The range of processed DDPTV images is a function of jet or fin wakes moving out of the sampling volume; only image sets with complete fin and jet wakes were processed. Approximately 75,000-125,000 particles were identified in each DDPTV image with triplet yields (matches of particles among the three cameras in the probe) of $\sim 50-60 \%$. Using a relaxation method for particle tracking (Pereira et al., 2006), approximately 18,000-25,000 particle vectors were obtained in the imaging volume. For interpolating the velocity vectors measured for the tracer particles onto a regular grid, Gaussian weighted interpolation was used with a voxel size of $16 \mathrm{~mm}$, percentage overlap of $75 \%$ and smoothing factor of 1.5 .

The laser illuminated the squid body brightly, which sometimes resulted in artificial body vectors in the vector field. Therefore, to address this issue, custom-designed masks were made in the 
INSIGHT 4G V3V software to remove the squid body from each image prior to DDPTV processing. The labor-intensive nature of custom mask development coupled with long processing times, particularly at the triplet matching step, made processing $>16$ DDPTV images per sequence impractical.

Velocity and vorticity fields were calculated using INSIGHT 4G V3V software and images were generated in Tecplot 360 (Tecplot, Bellevue, WA, USA). The data analysis followed the approach used in Bartol et al. (2016). Specifically, Matlab routines developed inhouse were used to compute impulse (I) associated with vortical flows generated by the squid using:

$$
\mathbf{I} / \rho=\frac{1}{2} \int_{V} \mathbf{x} \times \boldsymbol{\omega} \mathrm{d} V
$$

where $\mathbf{x}$ is the position vector, $\boldsymbol{\omega}$ is the vorticity vector $(\boldsymbol{\omega}=\nabla \times \mathbf{u}$ where $\mathbf{u}$ is the velocity vector), $\rho$ is the fluid density and the integral is computed over the volume of the vortex, $V$ (Saffman, 1992). The $3 \mathrm{D}$ volume surrounding the vortex of interest was selected using a graphical user interface (GUI) in Matlab, and the integral was computed over this volume to minimize the influence of measurement noise near the flow of interest. As impulse is the time integral of the force vector that generated the flow, the average thrust/lift vector (magnitude and 3D direction) was determined by dividing $\mathbf{I}$ by the period of the fin or jet cycle $(T)$. Time-averaged jet thrust $\left(\bar{F}_{\mathrm{jt}}\right)$ and fin thrust $\left(\bar{F}_{\mathrm{ft}}\right)$ were calculated using:

$$
\begin{gathered}
\bar{F}_{\mathrm{jt}}=-\mathbf{I} \cdot \hat{\mathbf{x}} / T, \\
\bar{F}_{\mathrm{ft}}=-\mathbf{I} \cdot \hat{\mathbf{x}} / T,
\end{gathered}
$$

where $\hat{\mathbf{x}}$ is the unit vector opposite the direction of tunnel flow and $T$ is the period of either the fin or jet cycle. The negative sign is included because Eqn 3 computes the fluid impulse and the impulse applied to the squid is in the opposite direction by Newton's Third Law. Similar to thrust, time-averaged lift forces from the jet $\left(\bar{F}_{\mathrm{jl}}\right)$ and fin $\left(\bar{F}_{\mathrm{fl}}\right)$ were computed as:

$$
\begin{gathered}
\bar{F}_{\mathrm{j} 1}=-\mathbf{I} \cdot \hat{\mathbf{y}} / T, \\
\bar{F}_{\mathrm{fl}}=-\mathbf{I} \cdot \hat{\mathbf{y}} / T,
\end{gathered}
$$

where $\hat{\mathbf{y}}$ is the unit vector in the vertical direction.

Total kinetic energy in the present study was computed using:

$$
\frac{E}{\rho}=\int \mathbf{u} \cdot(\mathbf{x} \times \boldsymbol{\omega}) \mathrm{d} V-\int\left[(\mathbf{u} \cdot \mathbf{x})(\mathbf{n} \cdot \mathbf{u})-\frac{1}{2} u^{2}(\mathbf{n} \cdot \mathbf{x})\right] \mathrm{d} S,
$$

which is mathematically equivalent to:

$$
E / \rho=\frac{1}{2} \int|\mathbf{u}|^{2} \mathrm{~d} V
$$

where $|\boldsymbol{u}|$ is the velocity magnitude, and $\mathrm{d} V$ and $\mathrm{d} S$ refer to volume and surface integrals, respectively, over the volume of the vortex and its corresponding bounding surface with outward unit normal vector $\mathbf{n}$. The advantage of this approach is that it will always give a positive value for $E$, but it does not necessarily isolate the $E$ associated with only the vortex of interest because of the influence of the surface integral term. Consequently, Eqn 6 provides a conservative measurement of the kinetic energy, i.e. the selected region can contain flow from neighboring vortices and any background noise in the selected region will increase the computed energy value. In application of Eqn 6, the background flow velocity was subtracted from the local velocity vector prior to computing the kinetic energy because only the excess kinetic energy is relevant for propulsive efficiency.

The rate of excess kinetic energy shed by the jet and fin $\left(E_{\mathrm{j}}\right.$ and $E_{\mathrm{f}}$, respectively) was computed by dividing $E$ by the appropriate jet or fin cycle. Propulsive efficiency $(\eta)$ was determined using the following equation:

$$
\eta=\frac{\bar{F}_{\mathrm{jt}} U+\bar{F}_{\mathrm{ft}} U}{\bar{F}_{\mathrm{jt}} U+\bar{F}_{\mathrm{ft}} U+E_{\mathrm{j}}+E_{\mathrm{f}}} .
$$

Researchers interested in the in-house Matlab code described above may contact the corresponding author.

\section{Statistical analysis}

We analyzed one representative fin sequence ( $\geq 3$ successive full fin cycles) in each of the 20 tail-first and 23 arms-first video clips. All statistical analysis was performed in SPSS v. 24 and all data were tested for normality using Shapiro-Wilk tests. Two-factor ANOVA were performed separately to assess differences in POD wavelength and frequency parameters (factors: swimming orientation and POD mode) and propulsor thrust and lift production (factors: swimming orientation and propulsor). One-way ANOVA (swimming orientation) were performed to assess differences in maximum fin amplitude location and propulsive efficiency. Tukey's HSD tests were used to analyze differences between means when $>2$ levels were considered. Linear regression analysis was performed to assess relationships between speed and the following variables: mode singular value (SV) ratios, fin beat frequency, fin amplitude, jet frequency and fin/jet frequency ratio. All means are reported \pm s.d.

\section{RESULTS \\ POD analysis}

The top three POD modes captured $86.8 \pm 0.02 \%$ of the total fin motion (range: $82.7-91.6 \%$ ). Given that modes $1-3$ explained the vast majority of the overall motion, we focused on these modes for this paper. Mode 1 was best characterized as a flapping motion while modes 2 and 3 were more wave-like (Figs 1 and 2). Specifically, modes 2 and 3 generally had at least one peak and one trough (which is incompatible with purely flapping motion) and these features were offset from one another in the two modes so that when modes 2 and 3 were combined with oscillating amplitudes slightly out of phase (as indicated in the amplitude plots), they tended to produce traveling wave motion. Evidence of both flap (up and down oscillation) and wave (sinusoidal oscillation) components were frequently visible in the fin sequences (Fig. 3A).

During tail-first swimming, mean $( \pm$ s.d. $)$ frequencies and wavelengths of modes 1,2 and 3 were $2.70 \pm 0.29 \mathrm{~Hz} / 1.22 \pm$ 0.16 fin lengths, $2.60 \pm 0.20 \mathrm{~Hz} / 1.22 \pm 0.24$ fin lengths and $4.58 \pm$ $0.32 \mathrm{~Hz} / 0.85 \pm 0.11$ fin lengths, respectively. During arms-first swimming, mean frequencies and wavelengths of modes 1,2 and 3 were $2.79 \pm 0.23 \mathrm{~Hz} / 1.26 \pm 0.15$ fin lengths, $2.61 \pm 0.28 \mathrm{~Hz} / 1.11 \pm$ 0.33 fin lengths and $3.36 \pm 0.28 \mathrm{~Hz} / 0.82 \pm 0.14$ fin lengths, respectively. No significant difference in frequencies or wavelengths was found between arms-first and tail-first swimming [2-factor ANOVA (frequency): swimming orientation $F_{1,120}=0.889, P=0.348$; 2-factor ANOVA (wavelength): swimming orientation $F_{1,118}=1.24$, $P=0.269]$. The other factor (POD mode) in the 2-factor ANOVA was significant for both frequency and wavelength [2-factor ANOVA (frequency): POD mode $F_{2,120}=7.08, P<0.001 ; 2$-factor ANOVA (wavelength): POD mode $\left.F_{2,118}=58.36, P<0.001\right]$, with Tukey HSD multiple comparison tests revealing that POD mode 3 was higher in frequency but lower in wavelength than POD modes 1 and 2 . 
A

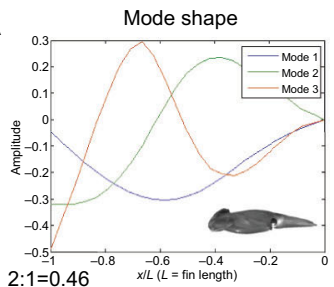

B

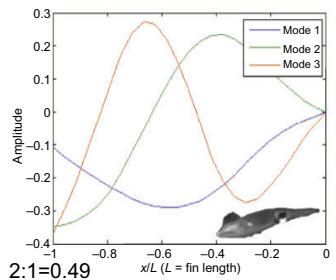

C

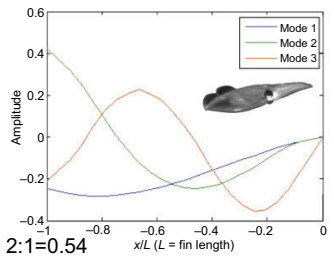

Mode amplitude
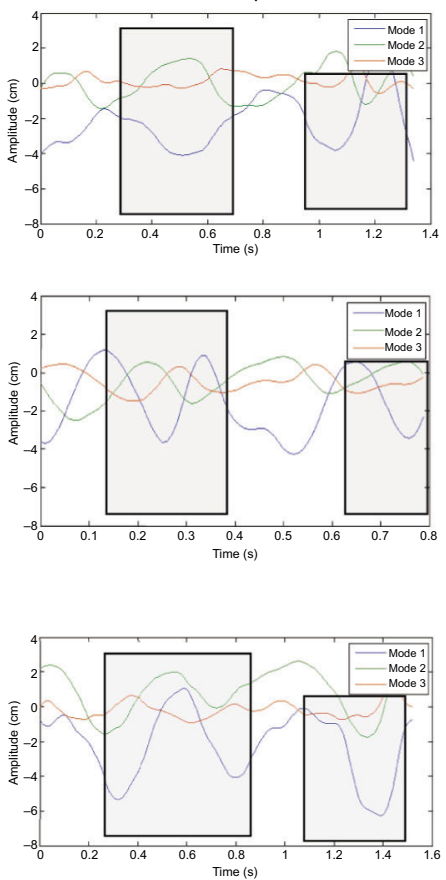

D

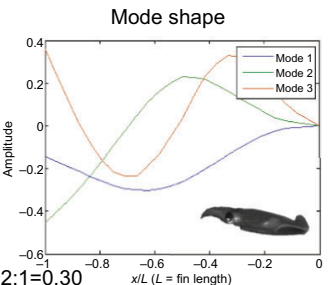

E
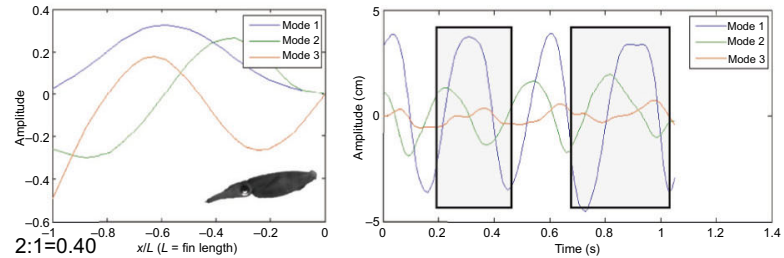

$\mathbf{F}$

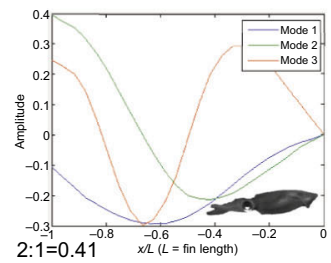

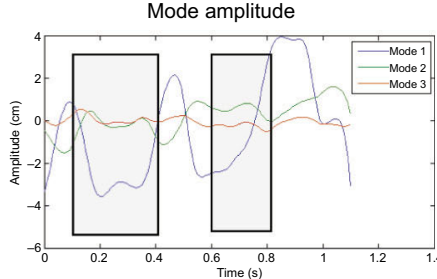

Mode amplitude

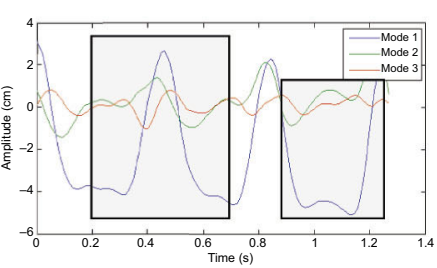

Fig. 2. Proper orthogonal decomposition (POD) results for the fin margin during squid swimming. (A-C) Arms-first swimming and (D-F) tail-first swimming. The mode shape insets demonstrate that mode 1 (blue) is more flap-like, while modes 2 and 3 (green and red) are more wave-like. The mode amplitude insets show that mode 1 has the highest amplitude over the time sequence. Although a range of motions was detected, the relative importance of modes 2 and 3 was generally higher for arms-first swimming than for tail-first swimming. This effect can be seen in the images above as mode 1 is typically greater relative to mode 2 in $D-F$ than in A-C, and mode 2:mode 1 singular value ratios are higher for arms-first (0.46-0.54) than tail-first (0.30-0.41) sequences. The shaded sections in the mode amplitude insets denote mantle contractions (jet pulses).

The maximum amplitude of mode 1 (flapping mode) occurred significantly farther from the leading edge of the fin during arms-first swimming $(64.8 \pm 6.1 \%$ of fin length) compared with tail-first swimming $(60.4 \pm 6.3 \%$ of fin length) (1-factor ANOVA: $\left.F_{1,36}=4.72, P=0.036\right)$.

On average, wave-like motions were more prominent during arms-first swimming than during tail-first swimming. This is reflected in significantly higher mode 2 :mode 1 and mode 3 :mode 1 $\mathrm{SV}$ ratios, i.e. ratio of wave motion relative to flapping motion, for arms-first swimming compared with tail-first swimming [2-factor ANOVA (swimming orientation): $F_{1,122}=14.29, P<0.001$; Fig. 2]. Across both swimming orientations, a significant difference in $\mathrm{SV}$ ratios was detected [2-factor ANOVA (ratio): $F_{2,122}=123.32$, $P<0.001]$. Tukey HSD multiple comparison tests revealed that the SV ratio of mode 3:mode 2 was greatest, followed by the SV ratio of mode 2 :mode 1 and mode 3:mode 1 .

As speed increased, fin motions became increasingly flap-like for both arms-first and tail-first swimming. This pattern can be seen in Fig. 4A,B,D,E, where the mode 2 :mode 1 and mode 3 :mode 1 amplitude ratios exhibit a linear decrease with speed [linear regressions (mode 2:mode 1): $P<0.03, R^{2}=0.16-0.26$; linear regressions (mode 3:mode 1): $\left.P<0.03, R^{2}=0.21-0.44\right]$. The importance of mode 3 relative to mode 2 (both wave-like motions) diminished with speed during tail-first swimming (linear regression, $P=0.01, R^{2}=0.31$ ), but not for arms-first swimming (linear regression, $P=0.56$; Fig. $4 \mathrm{C}, \mathrm{F}$ ). Fin beat frequency (mode 1 frequency) decreased with increased speed for both swimming orientations (linear regressions: $P<0.005, R^{2}=0.31-0.41$; Fig. 5A,D). During arms-first and tail-first swimming sequences, jet frequency (number of complete jet cycles per second) increased with increased speed (linear regression: $P<0.004, R^{2}=0.34-0.56$; Fig. 5B,E) and the ratio of fin-to-jet frequency decreased with speed (linear regressions: $P<0.006, \quad R^{2}=0.33-0.39$; Fig. 5C,F). No clear relationship was detected between fin amplitude and swimming speed in either the arms-first swimming orientation (linear regression: $P=0.547$ ) or tail-first orientation (linear regression: $P=0.358)$.

\section{DDPTV}

Vortex rings were observed in the wakes of both arms-first and tailfirst squid swimming. During arms-first swimming, linked or closely spaced vortices shed from the fins were common, with both downstroke vortices (vortex ring jet directed downward) and upstroke vortices (vortex ring jet directed upward) occurring within the wake chain (Fig. 6). More widely spaced vortex rings with no obvious linkage between upstrokes and downstrokes were also observed (Fig. 7A,C,D), as well as long cylindrical regions of concentrated vorticity with no discernible vortex ring structures (Figs 7B and 9C). These cylindrical regions tended to provide lift and stability as evident by downward- and rightward-directed flows in the fin wakes. During tail-first swimming, simple, widely spaced vortex rings (Figs 8A and 9A), sometimes with accompanying weaker regions of concentrated vorticity (Fig. 8C and 9D), were more common than more closely spaced/linked fin vortex structures (e.g. Fig. 8B,D). Fin wake complexity was generally greater for arms-first swimming than for tail-first swimming, including a greater prevalence of interconnected and closely spaced vortex structures, such as those in Fig. 6.

In some cases, fin vortex rings/structures were in close proximity to jet vortex rings, particularly for arms-first swimming instances 
A
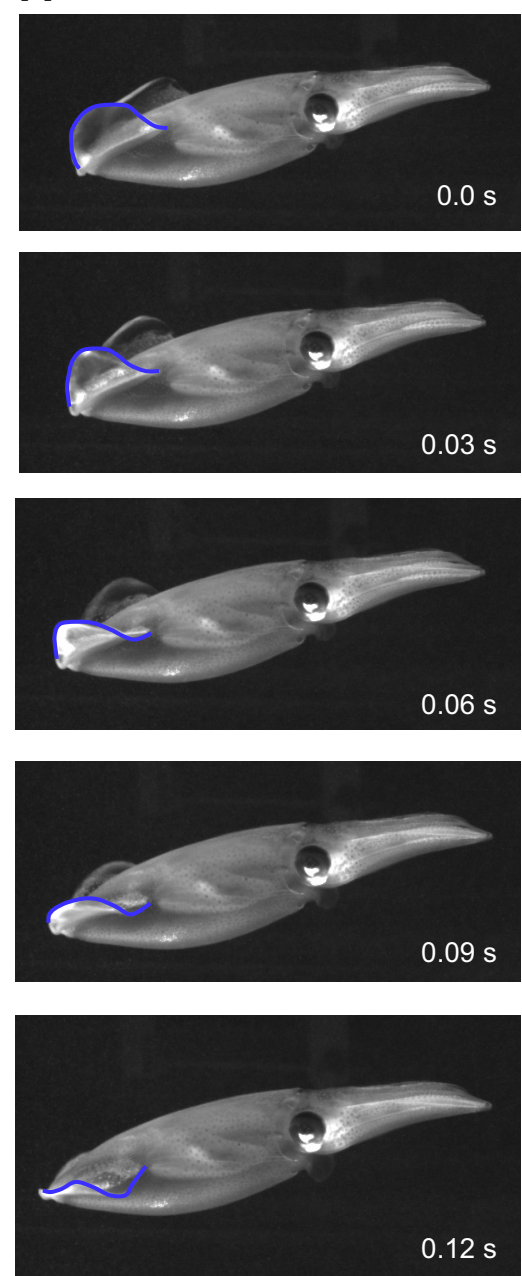
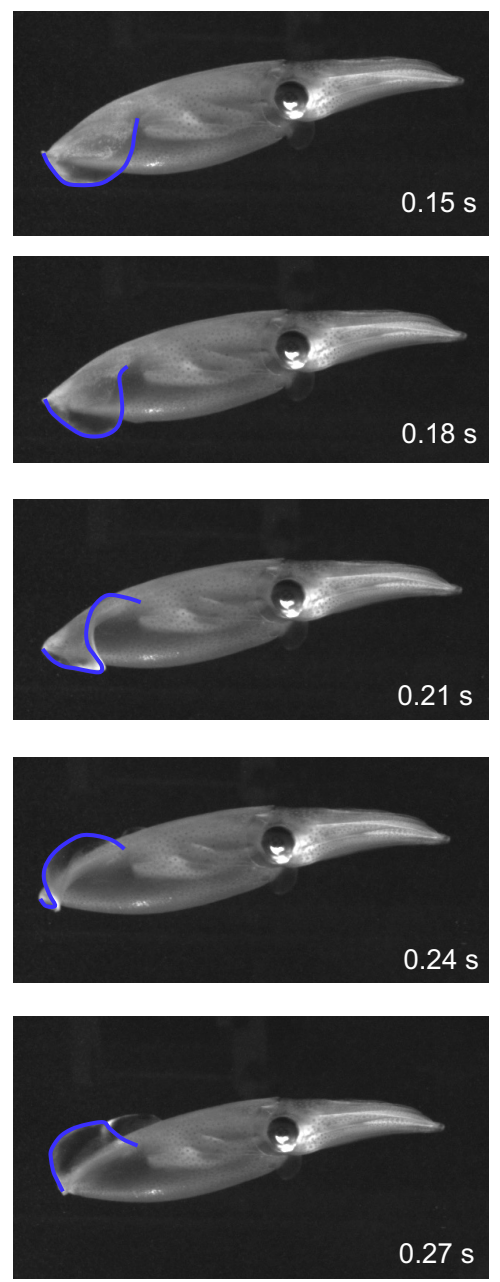

B
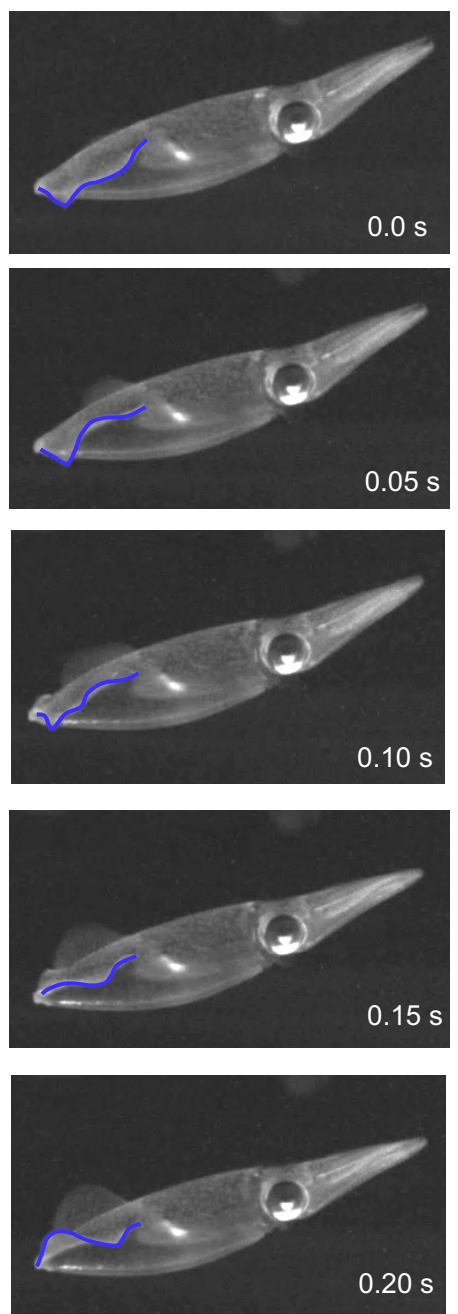

Fig. 3. Fin motions during a typical swimming cycle and a braking/stabilization sequence for arms-first swimming in a brief squid. (A) Typical swimming cycle and $(B)$ braking/stabilization sequence. Blue lines highlight the fin margin, while numbers in the lower right corner of each image show the time of each frame. Flapping (e.g. snapshot at $0.15 \mathrm{~s}$ ) and wave-like (snapshot at $0.21 \mathrm{~s}$ ) components are often present in fin sequences as shown in A. During braking/stabilization sequences, squid sometimes produce low-amplitude undulatory fin motions while keeping the overall angle of attack of the fin chord relatively constant (B).

(e.g. Figs 6D, 7B and 9C), though interactions were also observed in tail-first cases, especially when the animal was swimming at a low angle of attack (e.g. Fig. 8B). Sequences with higher mode 2: mode $1 \mathrm{SV}$ ratios (i.e. greater undulatory component) tended to correlate with greater fin complexity compared with those with lower mode 2:mode $1 \mathrm{SV}$ ratios. For example, the complex fin flows depicted in Fig. 6 for arms-first swimming all had mode 2:mode 1 SV ratios $\geq 0.44$, while simpler fin flows shown in Fig. 7 had mode 2 :mode 1 ratios $\leq 0.38$. A similar trend occurred for tail-first swimming, whereby the more complicated fin flows in Fig. 8B,D had higher mode 2 :mode 1 ratios $(0.40-0.41)$ than the simpler flows in Fig. 8A,C (0.27-0.30). High-speed flaps produced the simplest flow patterns. A common occurrence for both swimming orientations were pronounced fin braking/stabilizing wakes (drag) (Fig. 10). This stabilization wake was created by (1) holding the fin upward and/or broadside to oncoming flow (Fig. 10D-F) or (2) employing low amplitude undulations along the fin while keeping the overall fin chord angle relatively constant (Figs 3B and 10A-C) for brief periods in the fin cycle.

The Reynolds number $(R e)$ range for the animals considered in this study was 1000-15,000, which represents a subset of the broad $R e$ range $\left(10-10^{8}\right)$ encountered across squid taxa. The speed range considered for force and propulsive efficiency measurements was $0.42-3.20 \mathrm{DML} \mathrm{s}^{-1}\left(2.3-17.6 \mathrm{~cm} \mathrm{~s}^{-1}\right)$ for arms-first swimming and 0.53-4.79 DML s${ }^{-1}\left(3.3-26.4 \mathrm{~cm} \mathrm{~s}^{-1}\right)$ for tail-first swimming. During arms-first swimming, mean percentage fin contribution to thrust was $34.7 \pm 26.9 \%$, with a range of $0-79 \%$; mean percentage fin contribution to lift was 57.2 $\pm 33.8 \%$, with a range of $7-99 \%$. During tail-first swimming, mean percentage fin contribution to thrust was $31.9 \pm 26.3 \%$, with a range of $0-66 \%$; mean percentage contribution to lift was $29.5 \pm 25.5 \%$, with a range of $0-79.8 \%$.

The fins produced less thrust than the jet (mean fin thrust $=0.354$ $\pm 1.15 \mathrm{mN}$, mean jet thrust=2.19 $\pm 2.61 \mathrm{mN}$ ) [2-factor ANOVA (propulsor): $\left.F_{1,34}=8.10, P=0.007\right]$, with the fins sometimes producing braking forces (i.e. negative thrust; Fig. 10). However, no difference in thrust was detected between the two swimming orientations [2-factor ANOVA (orientation) $F_{1,34}=0.87, P=0.36$ ]. When lift forces were compared, a significant swimming orientation $\times$ propulsor interaction was detected [2-factor ANOVA (orientation $\times$ propulsor): $\left.F_{1,34}=4.42, P=0.043\right]$. This interaction was a product of the fins producing significantly less lift than the jet 
A

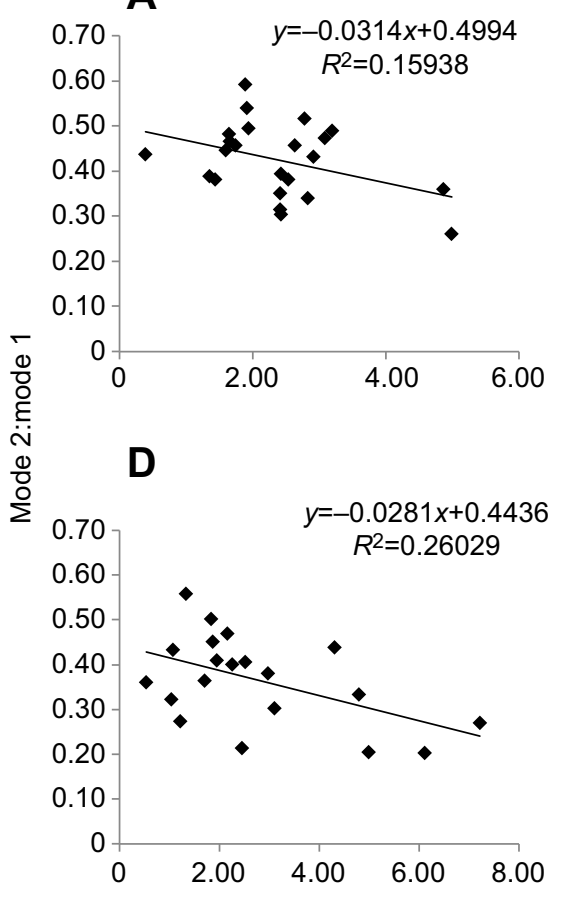

B

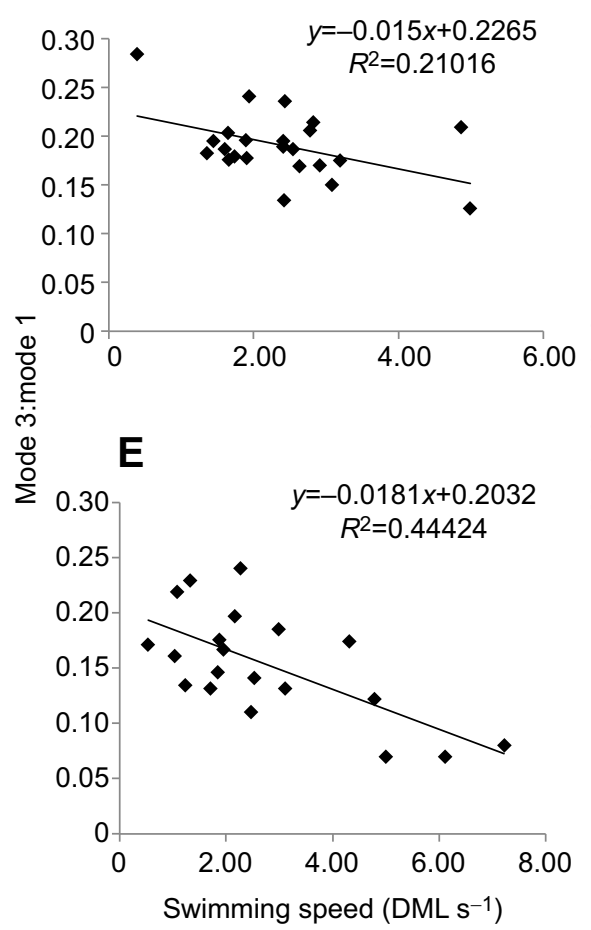

C
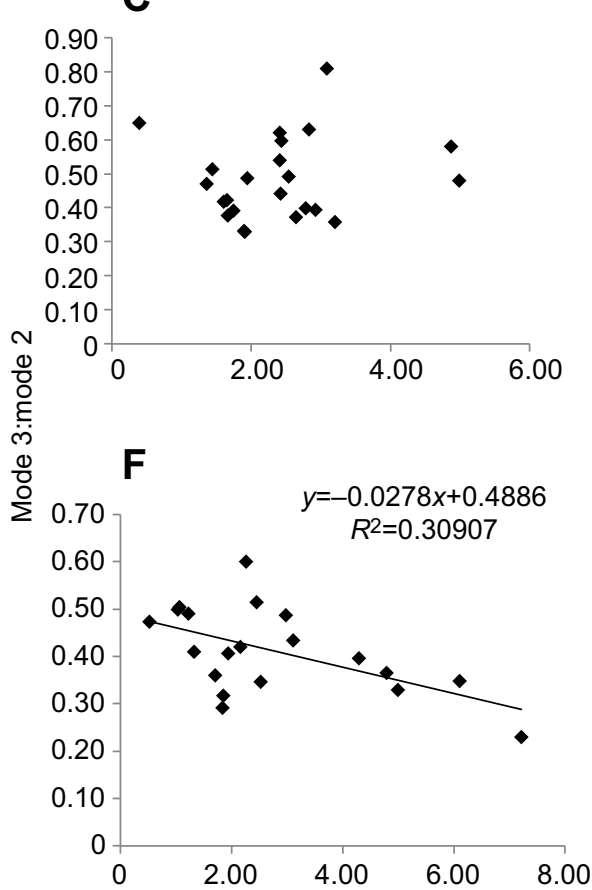

Fig. 4. Mode singular value (SV) ratios plotted as a function of squid swimming speed. (A-C) Arms-first swimming and (D-F) tail-first swimming. DML, dorsal mantle length.

during tail-first swimming, while the fins and jet produced similar levels of lift during arms-first swimming. As was the case with thrust, the fins produced negative lift at times, especially for sequences with pronounced upstrokes.
Mean propulsive efficiency was $61.8 \pm 18.5 \%$, with a range of $25.2-78.7 \%$. The fins improved propulsive efficiency by as much as $16 \%$ above the jet alone, but in some cases when the fins were braking, the fins lowered propulsive efficiency
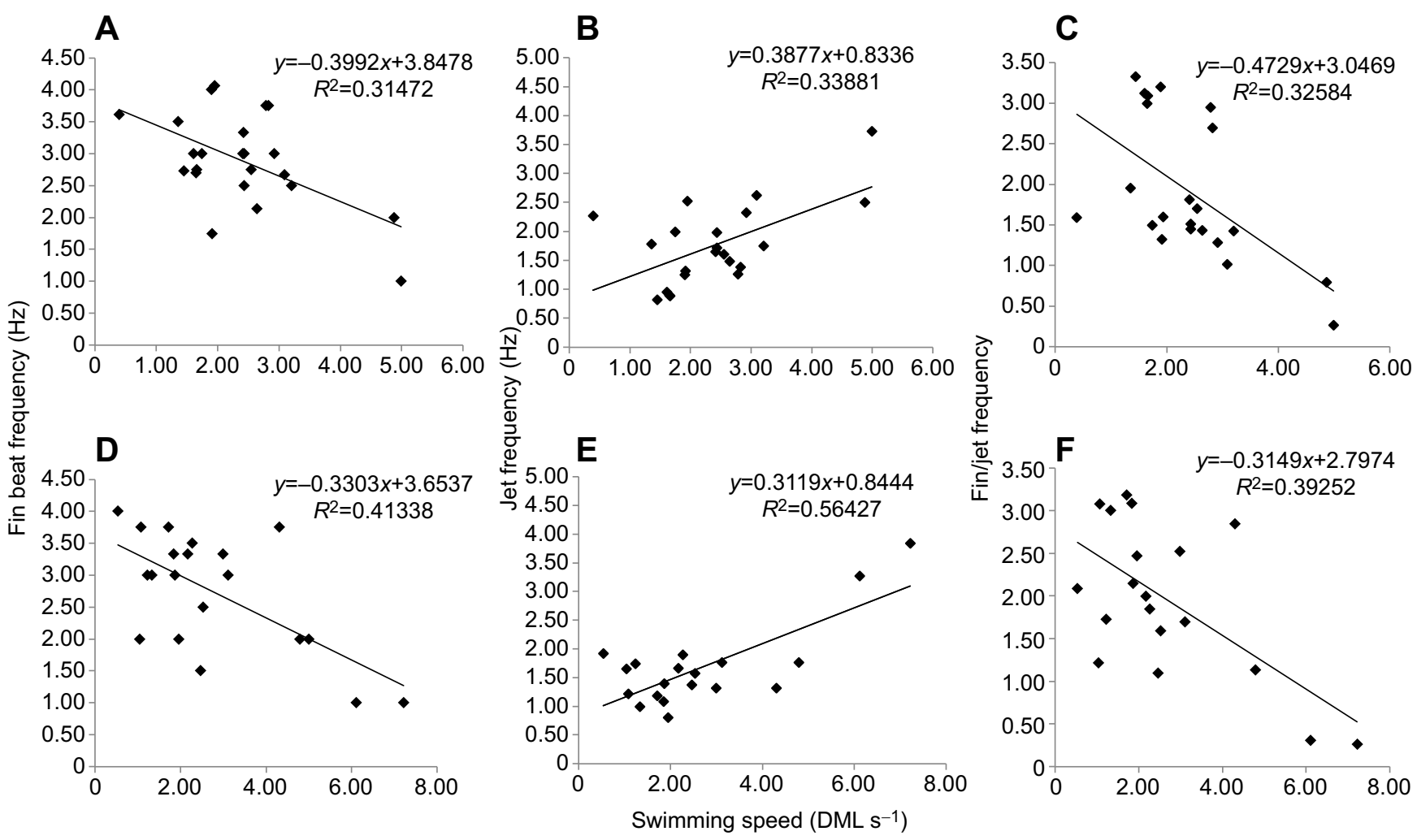

Fig. 5. Fin beat frequency, jet frequency and fin/jet frequency as a function of squid swimming speed. (A-C) Arms-first swimming and (D-F) tail-first swimming 

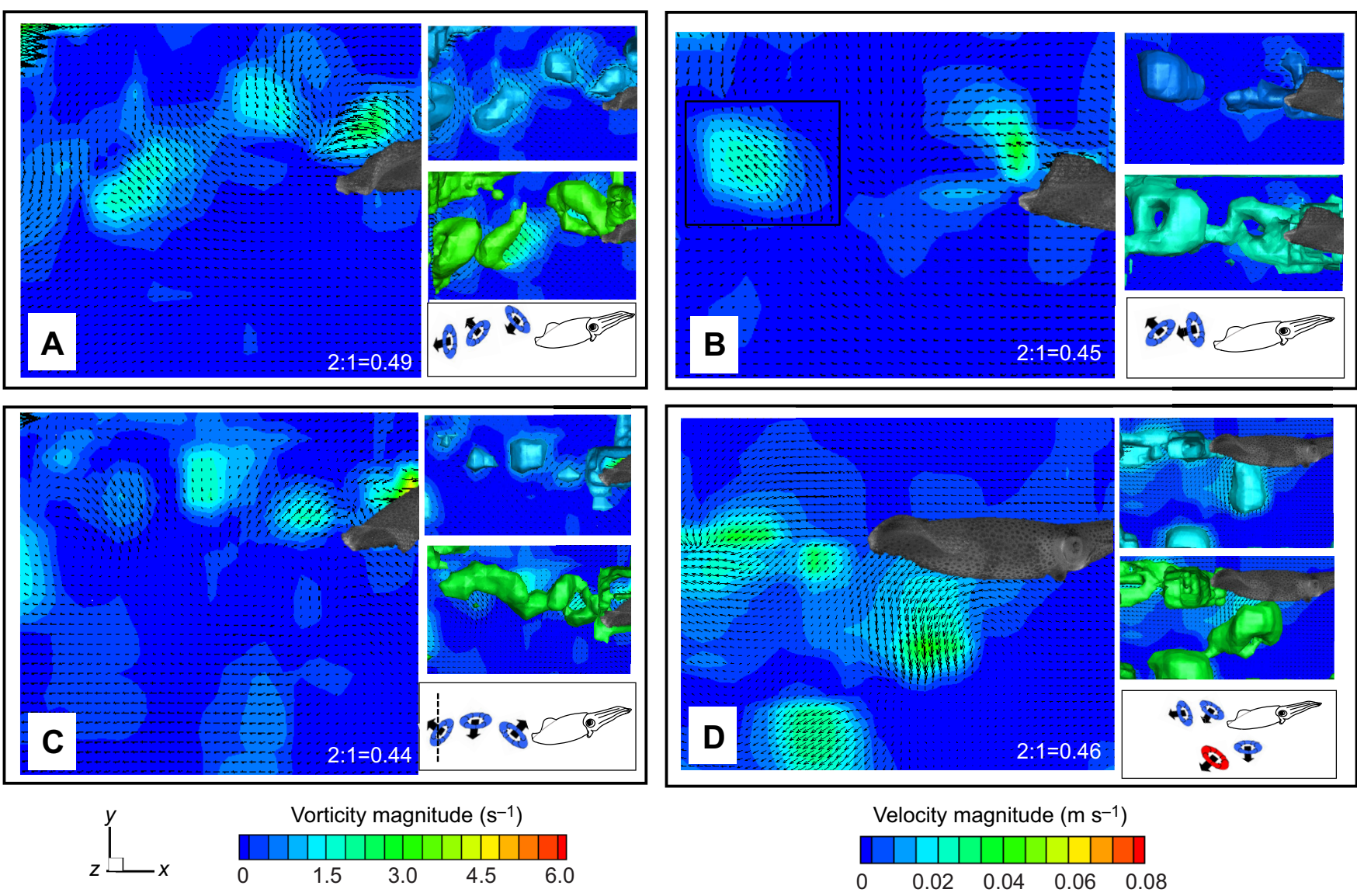

Fig. 6. Defocusing digital particle tracking velocimetry (DDPTV) images for brief squid swimming arms first with a high wave-like fin component. Velocity vector slices (left) and corresponding velocity magnitude isosurfaces (top right), vorticity magnitude isosurfaces (middle right) and schematic diagrams of vortex shedding (bottom right). Fin motions with prominent wave-like motions often produced chains of closely spaced vortex rings with upstroke flows (vortex rings with upward-directed jets) and downstroke flows (vortex rings with downward-directed jets). Each frame (A-D) reflects a separate swimming sequence and flows from the near-side fin are depicted for clarity, as the far-side fin produced similar flows in most cases. In B, an additional velocity vector slice (boxed region) is overlaid onto the larger velocity vector slice to illustrate flows from one of the vortices with a core that lies outside the depicted $z$-plane.

Background flow (tunnel speed) was subtracted from the images, with flow moving from right to left. In the schematic diagrams (bottom right), blue vortex rings are flows from the fin, red vortex rings are flows from the jet, and dashed lines show the edge of the frame. 2:1, mode 2:mode 1 singular value ratios from proper orthogonal decomposition (POD) analyses. The vorticity color bar applies to vorticity magnitude isosurfaces; the velocity color bar applies to both velocity slices and velocity magnitude isosurfaces.

by up to $29 \%$. No significant difference in propulsive efficiency was detected between arms-first sequences $(59.8 \pm 18.3 \%)$ and tail-first sequences $(65.4 \pm 19.1 \%)$ (1-factor ANOVA: $\left.F_{1,38}=0.82, P=0.37\right)$.

\section{DISCUSSION}

Although squid are frequently classified as jet-propelled swimmers, they use a hybrid propulsive system involving both a jet and fins. The role of the fins has been overlooked in many previous studies (as well as in the popular scientific literature), but they are integral to locomotion for numerous squid species. Indeed, the coordinated use of two hydrodynamically distinct modes makes squid an interesting intermediate group, falling between pure jet-propelled swimmers (e.g. jellyfish) and pure oscillatory/flapping swimmers (e.g. fish, turtles, mammals). In this study, we introduced two complementary approaches, i.e. POD and DDPTV, for assessing locomotion in squid, with an emphasis on the complex motions of fins. POD techniques have been used previously to understand the kinematics of highly deformable fish fins (Bozkurttas et al., 2006, 2009; Dong et al., 2010) and complex bat wing movements (Riskin et al., 2008), while DDPTV/3D velocimetry has been used to study swimming fish (Flammang et al., 2011a,b), squid (Bartol et al., 2016) and jellyfish (Gemmell et al., 2017). However, the present study is the first to combine POD techniques and DDPTV to study swimming in any aquatic animal.

\section{Fin motions}

Using POD, we found that both flap and wave-like components are present in the fin movements of brief squid L. brevis while swimming arms first and tail first. This is not surprising given that both flapping and undulatory fin patterns have been observed qualitatively in previous studies, including those focusing on L. brevis (Bartol et al., 2001b) and Doryteuthis (formerly Loligo) pealeii (Hoar et al., 1994; Anderson and DeMont, 2005). In prior studies, fin movements were characterized as either waves or flaps; however, the present study reveals that both components are present in fin movements, with flapping always being the dominant motion in L. brevis. While flapping is the dominant mode for both armsfirst and tail-first swimming, wave-like components are more pronounced during arms-first swimming than tail-first swimming based on our POD analysis. Maximum amplitude of the flap mode 

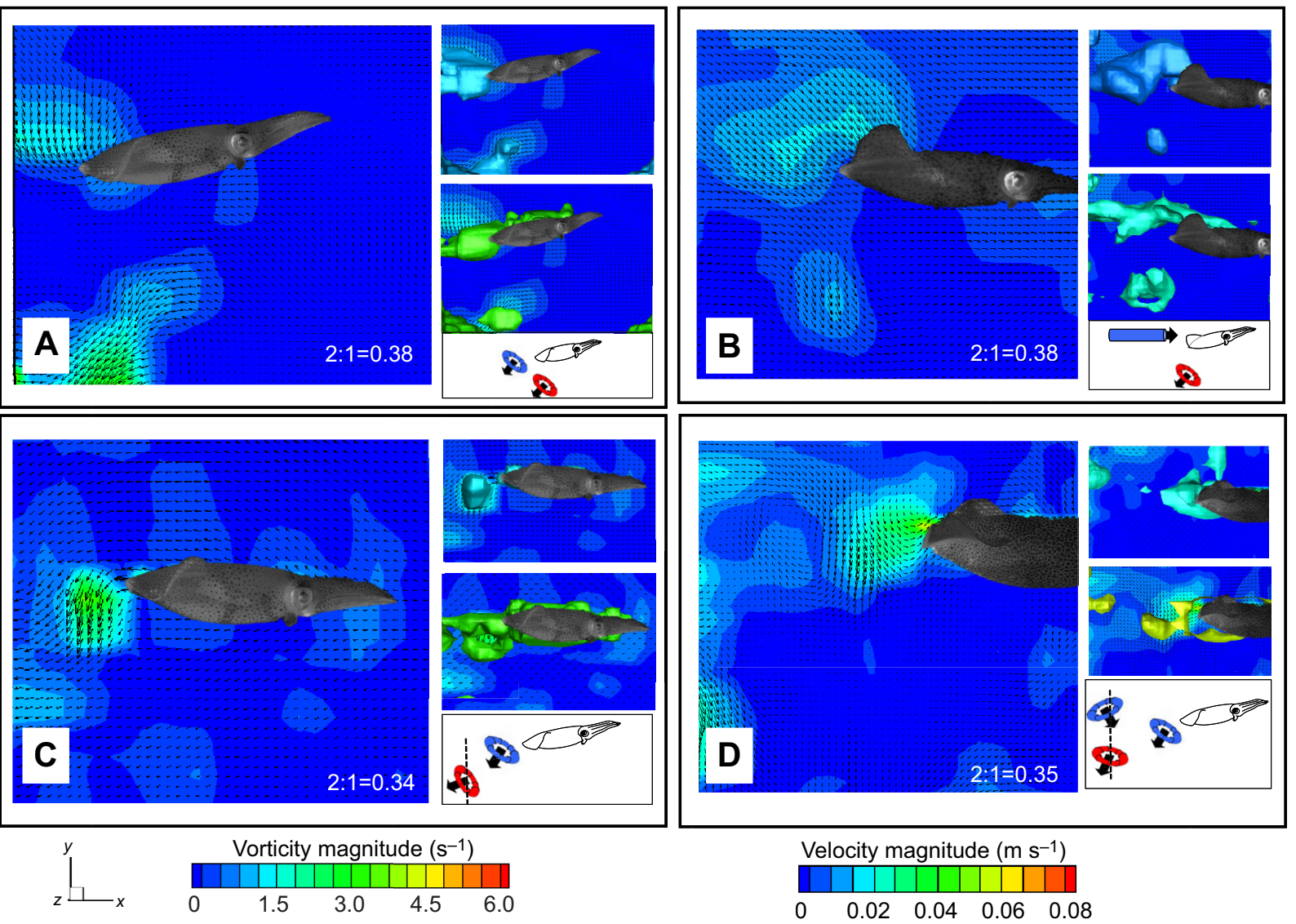

Fig. 7. DDPTV images for brief squid swimming arms first with a high flap-like fin component. Fin movements with less-prominent wave-like motions often produced simple, more spatially separated vortex rings $(A, C, D)$ or elongated trains of vorticity with no discernible upstroke and downstroke vortex rings (B). For figure layout description, see Fig. 6.

(mode 1) also occurred slightly farther back along the fin margin while swimming arms first versus tail first. Therefore, the fins are manipulated differently when they are at the trailing edge of the body (arms first) compared with when they are positioned at the leading edge of the body (tail first). Greater wave-like motions and a more downstream-positioned flap during arms-first swimming seemingly contribute to more lift generation, given higher observed lift forces for arms-first compared with tail-first swimming. This is not surprising considering that previous studies on fish and biorobotic systems showed that slight changes in fin behavior and mechanical properties can have a major influence on the timing, direction and magnitude of force production (Tytell et al., 2008; Tangorra et al., 2010; Neveln et al., 2014). Positioning the maximum fin amplitude farther downstream may also help counteract body pitching induced by rear-pointing jets.

Fin motions transition with swimming speed in L. brevis. For both swimming orientations, fin motions become more flap-like with increased swimming speed as evident by decreasing undulatory to flap mode ratios (i.e. mode 3 :mode 1 , mode 2: mode 1) with higher swimming speed. This finding is consistent with observations in L. brevis and D. pealeii, whereby fin motions were described as being more 'wave-like' or 'undulatory' at lower speeds (Bartol et al., 2001b; Anderson and DeMont, 2005). In fishes, rowing fin strokes (parallel to the direction of motion) and undulations ( $\geq 1$ propulsive wave present) are often used for efficient swimming at low speeds because they provide higher thrust, greater stability and better maneuverability than flapping fin strokes (perpendicular to the direction of motion or $<1 / 2$ propulsive wave present). At higher speeds, flapping motions are more prevalent because they can generate greater thrust via circulatory forces and are mechanically more efficient overall (Walker and Westneat, 2002; Di Santo and Kenaley, 2016; Di Santo et al., 2017). Therefore, the observed progression toward more flap-like fin motions at higher speeds is consistent with previous fish studies.

Fin beat frequency decreased, jet cycle frequency increased and the ratio of fin to jet frequency decreased with increasing swimming speed, indicating that the jet takes on more of the propulsive role at higher speeds. The increased propulsive role of the jet with speed has been shown previously in L. brevis (Bartol et al., 2001b, 2016). When kinematic data from Anderson and DeMont (2005) were pooled, decreases in fin beat frequency and the fin/jet frequency ratio with increased speed were also present for $D$. pealeii, though high variability in the data was present. High variability was also present in our study, particularly at intermediate speeds. At these speeds, the relationship between jet cycle frequency and speed is less clear, as consistent with other studies (Bartol et al., 2001b; Anderson and DeMont, 2005). This high variability may be a consequence of high propulsive flexibility at these intermediate speeds, where jet and fin forces can both be significant, allowing the squid to readily switch the relative force contributions of the two distinct systems. Bartol et al. (2001b) reported a decrease in fin beat frequency with increased swimming speed and an increase in jet cycle frequency with increased speed for some size classes of $L$. brevis swimming tail first. However, they found no clear fin or jet frequency relationship with swimming speed for arms-first 

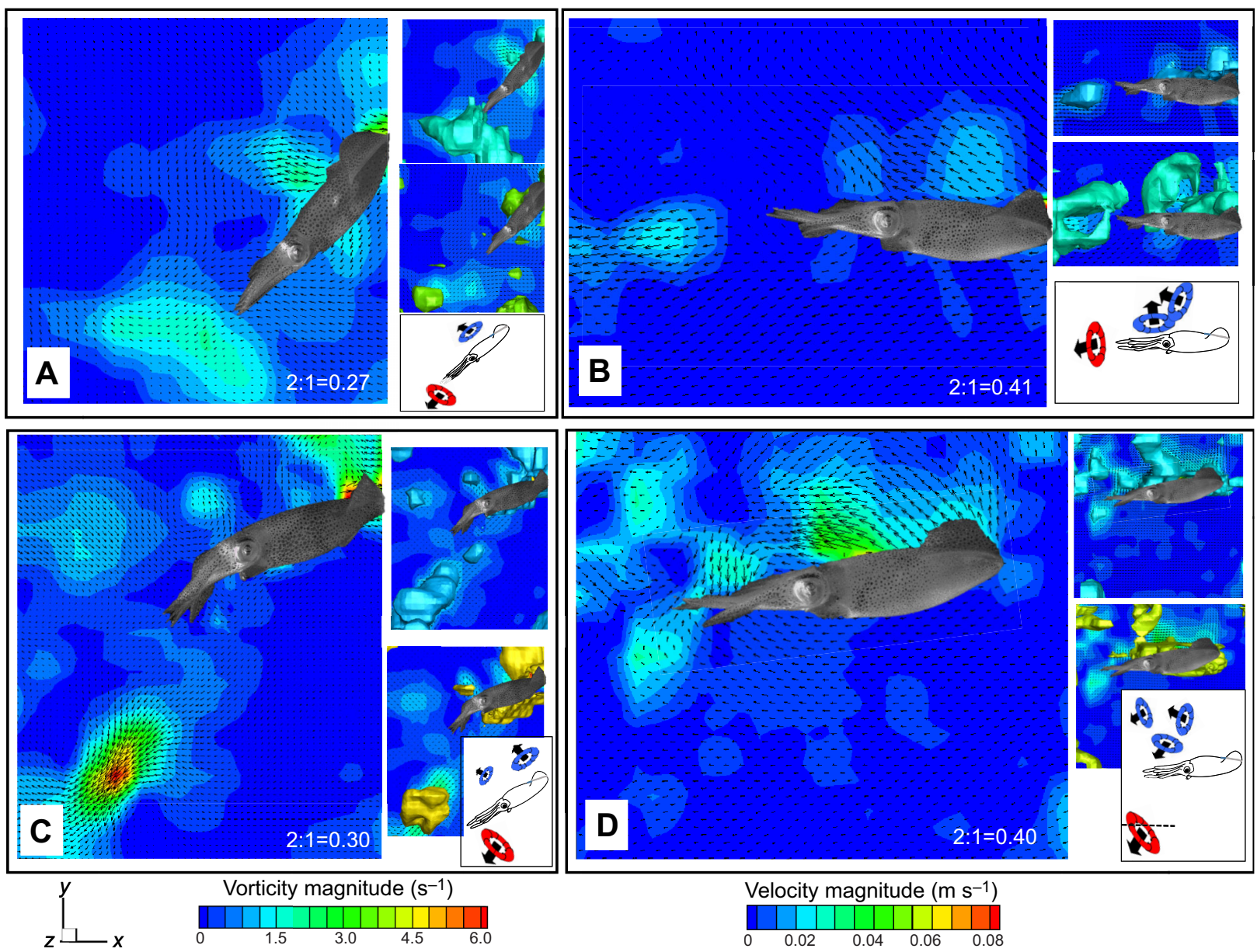

Fig. 8. DDPTV images for brief squid swimming tail first. Fin movements with less-prominent wave-like motions produced simple, widely spaced vortex rings (A,C) while fin movements with more-prominent wave-like motions produced more closely spaced vortex structures (B,D). For figure layout description, see Fig. 6 .

swimming. The absence of a relationship in arms-first swimming may derive from the consideration of lower speeds than the present study, in which higher swimming speed data had a significant impact on speed relationships.

Given the importance of fins for propulsion, why would fin beat frequency decrease with increased speed, especially at high speeds when thrust requirements are especially important? Limitations of fin muscle force-velocity relationships may be one reason. Fin movements are functions of the mechanical properties of the fin muscular hydrostat (Kier and Smith, 1985; Kier, 1989; Johnsen and Kier, 1993). As hydrodynamic-induced loading on the fins increases, presumably as swimming speed increases, the transverse muscles of the fins that provide power for fin flaps (Kier et al., 1989) are likely to respond with reduced shortening velocity. In the absence of recruitment of different fiber types (i.e. 'muscle gearing'; Rome, 2005), a possibility we have not yet investigated, or architectural gearing ratios (Brainerd and Azizi, 2005), high muscle force output and high muscle shortening velocities may be mutually exclusive in the fin muscles. The absence of a clear decrease in fin amplitude with increased swimming speed while fin beat frequency decreases provides some support for this hypothesis. Another possible reason for the observed decrease in fin frequency with increased speed is that fin propulsion may be less necessary at high speeds. As reported by Anderson and Grosenbaugh (2005) and Bartol et al. (2009b), jet slip is high at low speeds but decreases with increased speed. Reduced slip coupled with lower jet angles of attack contribute to higher propulsive jet efficiency at high speed, thereby lessening the need for fin propulsive input. Because hydrodynamic lift scales with the square of the swimming speed, lift generation by the fins is important at low speeds for negatively buoyant squid but less so at high speeds when significant lift contributions from the body and keeled arms are present. Thus, fins do not need to be as active at high speeds for lift production, which may be another reason for their decreased use at high speeds. Finally, heavy fin use at high speeds presumably incurs a high drag penalty and, consequently, it may be advantageous to rely more heavily on jet production at these speeds while keeping the fins close to the body over a greater part of the jet cycle.

\section{D fin flow patterns}

An important finding from this study is that greater undulatory components in fin motions correlate with more-complex flow features. In general, the wakes during arms-first swimming involved more interconnected vortex ring features than those during tail-first swimming, and greater vortex wake complexity was observed when undulatory mode to flapping mode SV ratios (mode 2:mode 1, mode 3:mode 1) were highest, irrespective of swimming orientation. In most cases, mode 2 :mode $1 \mathrm{SV}$ ratios $\leq 0.38$ resulted in more spatially separated vortex rings while mode 2 : mode $1 \mathrm{SV}$ ratios $>0.38$ resulted in more interconnected and closely spaced vortex ring patterns. Thus, it appears that pronounced undulatory components blur vortex ring separation and facilitate greater vortex ring merging, while more flap-like motions 


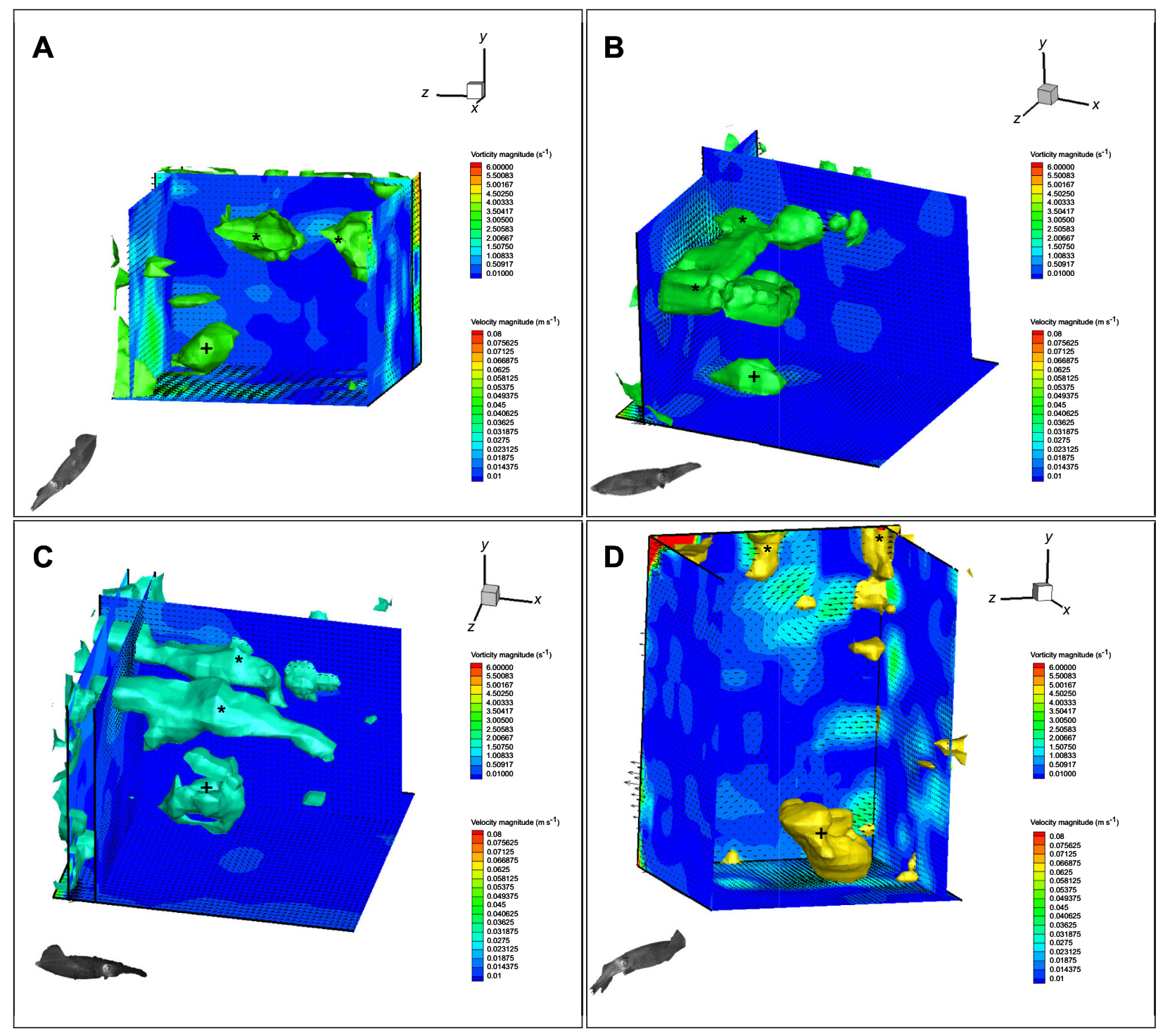

Fig. 9. DDPTV images for brief squid swimming showing spatial relationships between fin and jet flows. (A,D) Tail-first swimming and (B,C) arms-first swimming. A-D represent different viewing perspectives for flows in Figs $8 \mathrm{~A}, 7 \mathrm{~A}, 7 \mathrm{~B}$ and $8 \mathrm{C}$, respectively. In all cases, fin vortex rings/regions of concentrated vorticity from each fin $\left(^{*}\right)$ and jet vortex rings $(+)$ are visible. Background flow (tunnel speed) was subtracted from images. Squid images on the lower left of each panel represent the squid orientation when viewed laterally, but are not meant to indicate relative distance/relationship between the squid and the flows shown in the plots.

contribute to more spatially separated vortex ring patterns. Qualitative observations of fin motions by Stewart et al. (2010) are in agreement with the present study, whereby undulatory fin motions produced more continuously linked vortex chains. Although both closely spaced and more separated vortex ring patterns were observed over a wide range of speeds, as was also found by Stewart et al. (2010) using 2D particle image velocimetry, more pronounced undulatory fin movements with concomitant closely spaced vortex structures were most common at low and intermediate speeds. This may reflect greater flexibility in fin muscular hydrostatic function at these speeds when the muscle force-velocity tradeoffs are less significant. The closely spaced vortex patterns also presumably provide greater control of force magnitude and timing, which is beneficial at low/ intermediate speeds when maneuvering and lift/thrust adjustments are especially important compared with cruising at high speeds.
Use of the fins for trajectory adjustments was important based on the common occurrence of drag wakes behind the fins. Fin drag wakes were present in both swimming orientations (Fig. 10). The presence of drag wakes behind the fins is consistent with previous studies. In L. brevis, the fins can produce net drag for 38\% of armsfirst swimming speeds and $17 \%$ of tail-first swimming speeds (Stewart et al., 2010). Bartol et al. (2016) also found negative fin thrust (drag) for both swimming directions and suggested that because the fins are positioned at a considerable distance from the center of mass (relative to the jet), they can effect significant torque corrections. According to Weihs (2002), positioning fins at the leading edge is especially effective for stability adjustments; thus, the fins of squid may be especially important for stability when swimming tail first. Clearly, fin braking is integral for turning maneuvers, with kinematic studies showing that fin activity in $L$. brevis is important for tight and rapid turns (Jastrebsky et al., 2016) and for pursuing and capturing prey (Jastrebsky et al., 2017). Long 


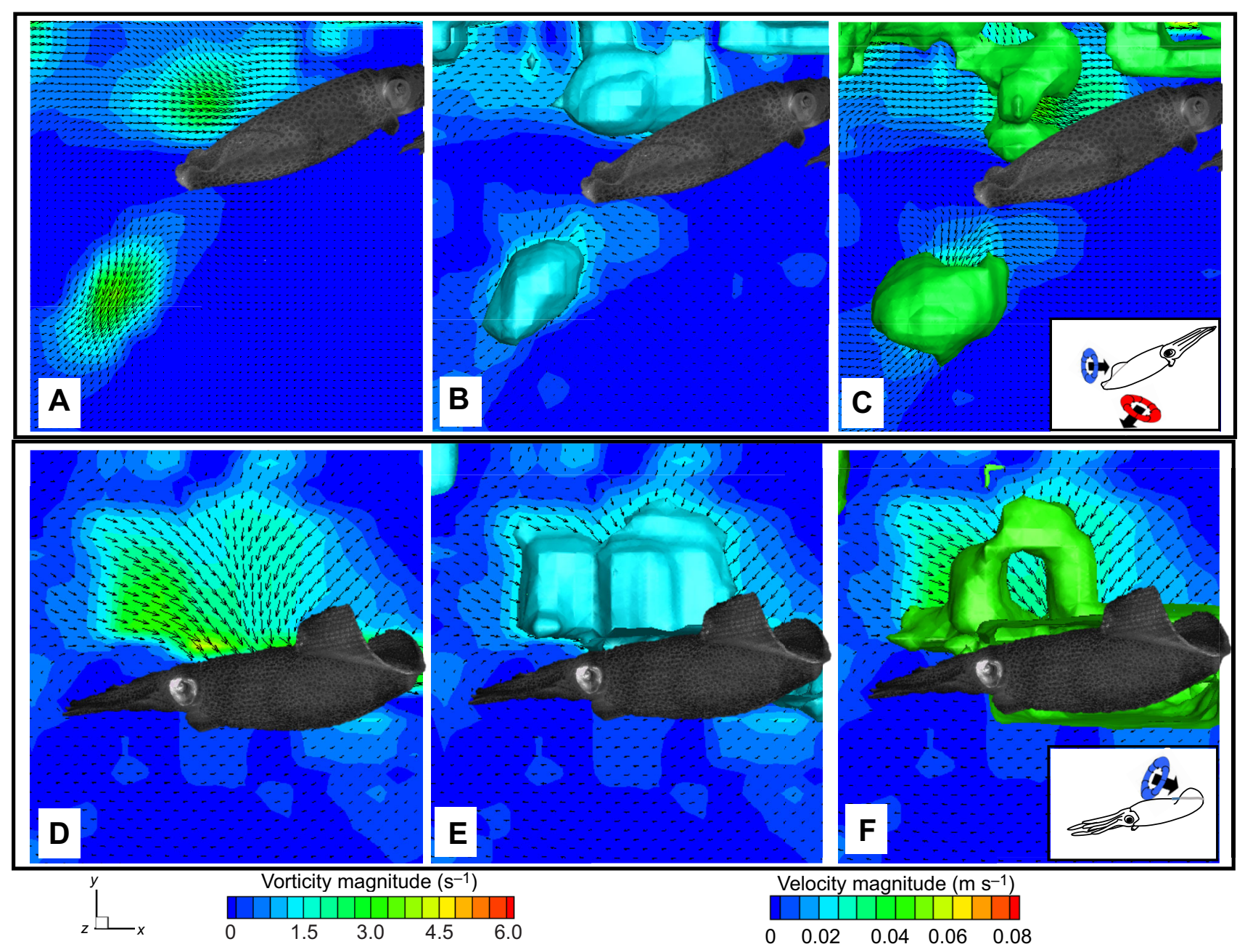

Fig. 10. DDPTV images for brief squid swimming arms first (top row) and tail first (bottom row) and using fin braking. Velocity slices (A,D), velocity magnitude isosurfaces $(B, E)$ and vorticity magnitude isosurfaces $(C, F)$ are shown. Flows from the near-side braking fin are depicted, with flows being directed opposite to oncoming flow (drag) and/or downward (lift). Background flow (tunnel speed) was subtracted from images, with flow moving from right to left. In the schematic insets, blue vortex rings are flows from the fin and red vortex rings are flows from the jet.

regions of concentrated vorticity that play lift generation and stability roles in L. brevis have been reported (Bartol et al., 2016). Similar patterns were observed in the current study (e.g. Figs 7B and 9C) and were often associated with a trajectory adjustment while the squid swam against the current in the water tunnel. The detection of fin drag/lift wakes in the present study provides further support for fins serving multiple functions beyond being propulsive thrust generators, including acting as lift producers, stabilizers and control surfaces, as suggested by others (Anderson and DeMont, 2005; Bartol et al., 2001a,b, 2008, 2009b, 2016; Hoar et al., 1994; O’Dor, 1988; O'Dor and Webber, 1991; Stewart et al., 2010; Webber and O’Dor, 1986).

In some cases, fin flows were close to jet flows (e.g. Figs 6D, 7B, $8 \mathrm{~B}$ and 9C). While fin kinematics and hydrodynamics were the primary focus of this experiment, the close proximity of fin and jet flows raises the possibility of interactive effects and hydrodynamic coordination between the two propulsive systems. Bartol et al. (2016) detected fin/jet flow interactions in L. brevis, especially at intermediate speeds $\left(1.5-3.0 \mathrm{DML} \mathrm{s}^{-1}\right)$, when the fins are highly active and the jet directs flow more in line with the fin wake. More horizontally directed jets are possible at intermediate swimming speeds versus low speeds for negatively buoyant squid because of reduced lift generation requirements (lift scales with the square of velocity). The majority of the speeds considered in the present study fall within the $1.5-3.0 \mathrm{DML} \mathrm{s}^{-1}$ swimming range; thus, the observed closely spaced fin/jet flows is not unexpected. Future work focusing on potential thrust augmentation associated with closely spaced/connected fin and jet flows would be valuable in advancing our understanding of coordination of the two propulsive systems.

\section{Fin gaits}

Anderson and DeMont (2005) identified two fin gait patterns in D. pealeii [15-30 cm mantle length (ML)] with a transition occurring at $1.4-1.8 \mathrm{ML} \mathrm{s}^{-1}$. For the lower speed gait, a downstroke occurs at the onset of jetting, an upstroke occurs during jetting, and a second downstroke coincides with jet closure. In this gait, the fin shape is wave-like. The higher speed gait involves a single flap (as opposed to a wave) at jet onset followed by fin wrapping for the remainder of the cycle. We observed a similar single flap and wrap pattern at the very highest speeds considered ( $>5 \mathrm{DML} \mathrm{s}^{-1}$ ), as well as a shift to more flap-like fin motions with increased speed as noted earlier. We have limited data at speeds exceeding $5 \mathrm{DML} \mathrm{s}^{-1}$ when this flap and wrap gait is prominent, as fin activity at sustained speeds above $5 \mathrm{DML} \mathrm{s}^{-1}$ 
is rare in L. brevis (Bartol et al., 2016). The higher speed switch to single flap and wrap behavior $\left(>5 \mathrm{DML} \mathrm{s}^{-1}\right)$ together with higher reported fin cycle frequencies $(4 \mathrm{~Hz}$ in L. brevis compared with $1.6 \mathrm{~Hz}$ in $D$. pealeii) suggest that fin activity is more integral to locomotion over a broader range of speeds in L. brevis compared with D. pealeii.

Using digital particle image velocimetry (DPIV), Stewart et al. (2010) identified four different fin wake patterns in L. brevis involving both isolated vortex rings and interconnected vortex ring structures. All four modes (I-IV) were identified in tail-first swimming, while two modes (II and III) were identified in arms-first swimming. During tail-first swimming, the most common modes observed in the present study resemble modes $\mathrm{III}_{\mathrm{T}}$ and IV in Stewart et al. (2010), where two spatially separated vortex rings are shed with each fin cycle (e.g. Fig. 8C) and a linked vortex structure is produced with each fin cycle (e.g. Fig. 8B,D), respectively. These two modes were also the most common modes observed by Stewart et al. (2010) for tail-first swimming in L. brevis. During arms-first swimming, the most common pattern observed in our study resembles mode $\mathrm{II}_{\mathrm{A}}$ in Stewart et al. (2010), which consists of a continuous chain of linked vortices (e.g. Fig. 6A-D). This mode was also the most frequent arms-first mode observed by Stewart et al. (2010). The less-common mode for arms-first swimming, mode $\mathrm{III}_{\mathrm{A}}$ in Stewart et al. (2010), which involved shed spatially separated vortex rings, was also detected in the present study (e.g. Fig. 7A,C,D).

The two least-frequent modes identified by Stewart et al. (2010) for tail-first swimming (modes $\mathrm{I}$ and $\mathrm{II}_{\mathrm{T}}$ ) were not observed in the present study. Given that mode I was detected at speeds $<0.5 \mathrm{DML} \mathrm{s}^{-1}$ (speeds not considered for tail-first swimming in the present study) and $\mathrm{II}_{\mathrm{T}}$ was only detected at a very narrow speed range (1.0-1.1 $\left.\mathrm{DML} \mathrm{s}^{-1}\right)$, it seems reasonable that these modes were not detected. Nonetheless, it should be noted that these flow patterns, and possibly others, may not have been resolved fully with the DDPTV technique. As mentioned in Bartol et al. (2016), our masking procedure, which involves removing all particles falling between the squid body and camera along the $z$-axis (camera viewing direction) to eliminate false flows driven by laser illumination of the body and fins, may have affected our quantification of flows coinciding with the squid body (as opposed to away from the body). This effect would only impact tail-first fin flows as the body is upstream of the fins during arms-first swimming, allowing for full resolution of shedding fin flows. Because all flows become fully visible downstream of the animals when flow development is complete, the force calculations, even those for tail-first swimming, are likely minimally affected by the masking procedure.

\section{Force and propulsive efficiency}

In general, the fins contributed less to overall thrust than the jet, and fin thrust contributions were similar for arms-first and tail-first swimming, providing 32-35\% of total thrust. While fin thrust was less than jet thrust on average, the fins provided as much as $79 \%$ of the total thrust for some swimming sequences. Bartol et al. (2008, 2016) found a similar maximum overall fin thrust contribution (83\%) for L. brevis using 2D and 3D velocimetry techniques. Using kinematic data and force calculations, Bartol et al. (2001b) predicted a maximum fin contribution of $55 \%$ of total thrust for L. brevis and Anderson and DeMont (2005) predicted a maximum fin contribution of $49 \%$ of total thrust for D. pealeii. Although the fins of some species of squid, such as Doryteuthis opalescens, do not appear to provide much propulsive force over the majority of their speed range (O'Dor, 1988), L. brevis relies on fin thrust propulsion over much of its sustained speed range, with the fins contributing more than the jet at times.

In addition to thrust production, the fins play an important role in lift generation in L. brevis. The fins contributed more lift during arms-first swimming (mean $\sim 57 \%$ of total lift; range $\sim 7-99 \%$ of total lift) than during tail-first swimming (mean $\sim 30 \%$ of total lift; range $\sim 0-80 \%$ of total lift), with lift contribution levels being statistically similar between the fins and jet in the arms-first mode. The reason for greater reliance on the fins for lift during arms-first swimming is not known, but perhaps reduced fin flow/body interactions afforded by rearward positioning of the fins allows for greater lift generation, or perhaps the squid generate less hydrodynamic lift from flow over the arms and body, and thus they need greater lift production from the fins.

Mean propulsive efficiency was $62 \%$, with efficiencies reaching a maximum of $79 \%$ for the speed range considered in this study. The mean propulsive efficiency reported here agrees well with Bartol et al. (2009b, 2016), who reported mean propulsive efficiencies of $64-66 \%$, but is lower than that reported in a more limited L. brevis dataset (78\%) (Bartol et al., 2008). The maximum propulsive efficiency reported in the current study is lower than the $96 \%$ reported by Bartol et al. (2016) and 97\% reported by Anderson and Grosenbaugh (2005), but these high-efficiency values were observed at swimming speeds exceeding those considered here, where the focus was intermediate speeds with heavy fin use. As shown by Anderson and Grosenbaugh (2005) and Bartol et al. (2009b, 2016), propulsive efficiencies increase with increased swimming speed.

Previous studies have shown that fin activity improves propulsive efficiency by as much as 10-19\% (Bartol et al., 2008, 2016) over the jet alone, which is consistent with our maximum finding of $16 \%$ above the jet alone. Just as Bartol et al. (2016) found that the fins can also lower propulsive efficiency when braking (by as much as $11 \%$ ), we found that propulsive efficiency can be lowered by as much as $29 \%$ when fin contributions are considered with the jet. Reduced propulsive efficiency in some sequences is likely the price paid for fin-enhanced stabilization, lift generation and maneuvering - behaviors that contribute to excess kinetic energy in the wake. The large range of fin effects on propulsive efficiency reflects the multiple roles of fins, shifting in some cases from propulsors to rudders/stabilizers/wings.

The wide repertoire of fin motions coupled with vectored jetting provides great flexibility in swimming. The two propulsive systems allow squid to swim arms first or tail first without difficulty. In fact, squid are so adept at adjusting to different conditions (e.g. degree of funnel bending, fin positioning, arm positioning, etc.) in the two orientations that they can maintain similar propulsive efficiency for arms-first $(\sim 60 \%)$ and tail-first $(\sim 65 \%)$ swimming. The lack of a significant difference in propulsive efficiency between the two orientations is consistent with Bartol et al. (2016).

\section{Concluding remarks}

Studying fluid-structure interactions in highly flexible systems is not trivial. This is especially true for squid, which swim using coordinated movements of highly flexible, muscular hydrostatic fins and a pulsed vectored jet. Given the complexity of the flow fields and fin movements of squid, we employed an integrated approach using POD and DDPTV to decouple fin motions and understand their momentum consequences. Our results demonstrate that both flap and wave components are always present during fin movements, but the relative importance of wave-like motions 
changes with swimming orientation, i.e. arms first versus tail first, and swimming speed. In general, fin movements with greater relative wave-like motions produce more complex interconnected vortex ring wakes, while fin movements dominated by flapping result in more spatially separated vortex ring patterns. The highly flexible nature of squid fins facilitates the production of a suite of different wake patterns with beneficial momentum imparting effects. Although the jet often produces the majority of the thrust, our results show that the fins can contribute more thrust than the jet at times and consistently produce comparable levels of lift to those of the jet in the arms-first mode. The fins also produce significant drag signatures that can aid squid in stabilization and maneuvering. Clearly, the versatile fins play multiple roles in squid locomotion, including boosting overall propulsive efficiency, and when coupled with the jet, allow squid to perform a wide range of swimming behaviors that are integral to their ecological success.

\section{Acknowledgements}

We especially thank Rachel Jastrebsky for assistance during animal capture and maintenance, data collection and processing. Brenna Perez and Darryl Fox assisted in the digitization of fin margins. We also thank the staff at the Virginia Institute of Marine Science Eastern Shore Laboratory for field assistance and TSI, Inc. (Shoreview, MN, USA) for providing an additional software key for processing.

\section{Competing interests}

The authors declare no competing or financial interests.

\section{Author contributions}

Conceptualization: I.K.B., P.S.K., J.T.T.; Methodology: I.K.B., P.S.K.; Software: P.S.K.; Formal analysis: I.K.B., C.A.Y.; Writing - original draft: I.K.B.; Writing - review \& editing: I.K.B., P.S.K., C.A.Y., J.T.T.; Supervision: I.K.B.; Project administration: I.K.B.; Funding acquisition: I.K.B., P.S.K., J.T.T.

\section{Funding}

This project was supported by the National Science Foundation (IOS 1115110 and IOS 1557669 to I.K.B., P.S.K. and J.T.T.).

\section{References}

Anderson, E. J. and DeMont, M. E. (2000). The mechanics of locomotion in the squid Loligo pealei: locomotory function and unsteady hydrodynamics of the jet and intramantle pressure. J. Exp. Biol. 203, 2851-2863.

Anderson, E. and DeMont, M. E. (2005). The locomotory function of the fins in the squid Loligo pealei. Mar. Freshw. Behav. Physiol. 38, 169-189.

Anderson, E. J. and Grosenbaugh, M. (2005). Jet flow in steadily swimming adult squid. J. Exp. Biol. 208, 1125-1146.

Bartol, I. K., Mann, R. and Patterson, M. R. (2001a). Aerobic respiratory costs of swimming in the negatively buoyant brief squid Lolliguncula brevis. J. Exp. Biol. 204, 3639-3653.

Bartol, I. K., Patterson, M. R. and Mann, R. (2001b). Swimming mechanics and behavior of the shallow-water brief squid Lolliguncula brevis. J. Exp. Biol. 204, 3655-3682.

Bartol, I. K., Krueger, P. S., Thompson, J. T. and Stewart, W. J. (2008). Swimming dynamics and propulsive efficiency of squids throughout ontogeny. Integr. Comp. Biol. 48, 720-733.

Bartol, I. K., Krueger, P. S., Stewart, W. J. and Thompson, J. T. (2009a). Pulsed jet dynamics of squid hatchlings at intermediate Reynolds numbers. J. Exp. Biol. 212, 1506-1518.

Bartol, I. K., Krueger, P. S., Stewart, W. J. and Thompson, J. T. (2009b) Hydrodynamics of pulsed jetting in juvenile and adult brief squid Lolliguncula brevis: evidence of multiple jet "modes" and their implications for propulsive efficiency. J. Exp. Biol. 212, 1889-1903.

Bartol, I. K., Krueger, P. S., Jastrebsky, R. A., Williams, S. and Thompson, J. T. (2016). Volumetric flow imaging reveals the importance of vortex ring formation in squid swimming tail-first and arms-first. J. Exp. Biol. 219, 392-403.

Bozkurttas, M., Dong, H., Mittal, R., Madden, P. and Lauder, G. V. (2006) Hydrodynamic Performance of Deformable Fish Fins and Flapping Foils, Proc. 44th AIAA Aerospac Sciences Meeting and Exhibit, Jan. 9-12, Reno, NV (Paper no. AIAA 2006-1392)

Bozkurttas, M., Mittal, R., Dong, H., Lauder, G. V. and Madden, P. (2009). Low- dimensional models and performance scaling of a highly deformable fish pectoral fin. J. Fluid Mech. 631, 311-342.
Brainerd, E. L. and Azizi, E. (2005). Muscle fiber angle, segment bulging and architectural gear ratio in segmented musculature. J. Exp. Biol. 208, 3249-3261.

Crandell, K. E. and Tobalske, B. W. (2015). Kinematics and aerodynamics of avian upstrokes during slow flight. J. Exp. Biol. 218, 2518-2527.

Di Santo, V. and Kenaley, C. P. (2016). Skating by: low energetic costs of swimming in a batoid fish. J. Exp. Biol. 219, 1804-1807.

Di Santo, V., Blevins, E. L. and Lauder, G. V. (2017). Batoid locomotion: effects of speed on pectoral fin deformation in the little skate, Leucoraja erinacea. J. Exp. Biol. 220, 705-712

Dong, H., Bozkurttas, M., Mittal, R., Madden, P. and Lauder, G. V. (2010) Computational modelling and analysis of the hydrodynamics of a highly deformable fish pectoral fin. J. Fluid Mech. 645, 345-373.

Flammang, B. E., Lauder, G. V., Troolin, D. R. and Strand, T. E. (2011a) Volumetric imaging of fish locomotion. Biol. Lett. 7, 695-698.

Flammang, B. E., Lauder, G. V., Troolin, D. R. and Strand, T. (2011b). Volumetric imaging of shark tail hydrodynamics reveals a three-dimensional dual-ring vortex wake structure. Proc. Biol. Sci. 278, 3670-3678.

Gemmell, B. J., Troolin, D. R., Costello, J. H., Colin, S. P. and Satterlie, R. A. (2017). Control of vortex rings for manoeuvrability. J. R. Soc. Interface. 12 , 20150389

Hanlon, R. T., Hixon, R. F. and Hulet, W. H. (1983). Survival, growth and behavio of the loliginid squids Loligo plei, Loligo pealei, and Lolliguncula brevis (Mollusca: cephalopoda) in closed sea water systems. Biol. Bull. 165, 637-685.

Hoar, J. A., Sim, E., Webber, D. M. and O'Dor, R. K. (1994). The role of fins in the competition between squid and fish. In Mechanics and Physiology of Animal Swimming (ed. L. Maddock, Q. Bone and J. M. V. Rayner), pp. 27-33. Cambridge: Cambridge University Press.

Jastrebsky, R. A., Bartol, I. K. and Krueger, P. S. (2016). Turning performance in squid and cuttlefish: unique dual mode, muscular hydrostatic systems. J. Exp. Biol. 219, 1317-1326.

Jastrebsky, R. A., Bartol, I. K. and Krueger, P. S. (2017). Turning performance of brief squid Lolliguncula brevis during attacks on shrimp and fish. J. Exp. Biol. 220, 908-919.

Johnsen, S. and Kier, W. M. (1993). Intramuscular crossed connective tissue fibres: skeletal support in the lateral fins of squid and cuttlefish (Mollusca: Cephalopoda). J. Zool. Lond. 231, 311-338.

Johnson, W., Soden, P. D. and Trueman, E. R. (1972). A study in jet propulsion: an analysis of the motion of the squid, Loligo Vulgaris. J. Exp. Biol. 56, 155-165.

Kier, W. M. (1989). The fin musculature of cuttlefish and squid (Mollusca Cephalopoda): morphology and mechanics. J. Zool. 217, 23-38.

Kier, W. M. and Smith, K. K. (1985). Tongues, tentacles and trunks. J. Linn. Soc Lond. Zool. 83, 23-38

Kier, W. M. and Thompson, J. T. (2003). Muscle arrangement, function and specialization in recent coleoids. Berliner Palaobiologische Abhandlungen 03, 141-162

Kier, W. M., Smith, K. K. and Miyan, J. A. (1989). Electromyography of the fin musculature of the cuttlefish Sepia officinalis. J. Exp. Biol. 143, 17-31.

Lentink, D. and Dickinson, M. H. (2009). Biofluiddynamic scaling of flapping, spinning and translating fins and wings. J. Exp. Biol. 212, 2691-2704.

Marple, S. L. (1987). Digital Spectral Analysis, pp. 373-378. Englewood Cliffs, NJ Prentice-Hall.

Muramatsu, K., Yamamoto, J., Abe, T., Sekiguchi, K., Hoshi, N. and Sakurai, Y (2013). Oceanic squid do fly. Mar. Biol. 160, 1171-1175.

Neveln, I. D., Bale, R., Bhalla, A. P. S., Curet, O. M., Patankar, N. A. and Maclver, M. A. (2014). Undulating fins produce off-axis thrust and flow structures. J. Exp. Biol. 217, 201-213.

O'Dor, R. K. (1988). The forces acting on swimming squid. J. Exp. Biol. 442, 421-442.

O'Dor, R. K. and Webber, D. M. (1991). Invertebrate athletes: trade-offs between transport efficiency and power density in cephalopod evolution. J. Exp. Biol. 160, 93-112

O'Dor, R. K. Hoar, J. A., Webber, D. M., Carey, F. G., Tanaka, S., Martins, H. and Porteiro, F. M. (1995). Squid (Loligo forbesi) performance and metabolic rates in nature. Mar. Behav. Phys. 25, 163-177

O'Dor, R., Stewart, J., Gilly, W., Payne, J., Borges, T. C. and Thys, T. (2013) Squid rocket science: How squid launch into air. Deep Sea Res. Part II: Tropical Stud. Ocean., 95, 113-118.

Pereira, F., Stüer, H., Graff, E. C. and Gharib, M. (2006). Two-frame 3D particle tracking. Meas. Sci. Technol. 17, 1680-1692.

Riskin, D. K., Willis, D. J., Iriarte-Díaz, J., Hedrick, T. L., Kostandov, M., Chen, J., Laidlaw, D. J., Breuer, K. and Swartz, S. M. (2008). Quantifying the complexity of bat wing kinematics. J. Theor. Biol. 254, 604-615.

Rome, L. C. (2005). Principles of actuation in the muscular system of fish. IEEE J. Oceanic Eng. 30, 630-646.

Saffman, P. G. (1992). Vortex Dynamics. Cambridge: Cambridge University Press. Stewart, W. J., Bartol, I. K. and Krueger, P. S. (2010). Hydrodynamic fin function of brief squid, Lolliguncula brevis. J. Exp. Biol. 213, 2009-2024.

Tangorra, J. L., Lauder, G. V., Hunter, I. W., Mittal, R., Madden, P. G. A. and Bozkurttas, M. (2010). The effect of fin ray flexural rigidity on the propulsive 
forces generated by a biorobotic fish pectoral fin. J. Exp. Biol. 213, 4043-4054.

Tytell, E. D., Standen, E. M. and Lauder, G. V. (2008). Escaping flatland: three- dimensional kinematics and hydrodynamics of median fins in fishes. J. Exp. Biol. 211, 187-195.

Vecchione, M. and Roper, C. (1991). Cephalopods observed from submersibles in the western North Atlantic. Bull. Mar. Sci. 49, 433-445.

Vecchione, M., Young, R. E., Guerra, A., Lindsay, D. J., Clague, D. A., Bernhard, J. M., Sager, W. W., Gonzalez, A. F., Rocha, F. J. and Segonzac, M. (2001). Worldwide observations of remarkable deep-sea squids. Science 294, 2505-2506.

Vecchione, M., Roper, C. E., Widder, E. A. and Frank, T. M. (2002). In situ observations on three species of large-finned deep-sea squids. Bull. Mar. Sci. 71, 893-901.
Webber, D. M. and O'Dor, R. K. (1986). Monitoring the metabolic rate and activity of free-swimming squid with telemetered jet pressure. J. Exp. Biol. 126, 205-224.

Weihs, D. (2002). Stability versus maneuverability in aquatic locomotion. Integr. Comp. Biol. 42, 127-134.

York, C. A. and Bartol, I. K. (2014). Lateral line analogue aids vision in successful predator evasion for the brief squid, Lolliguncula brevis. J. Exp. Biol. 217, 2437-2439.

York, C. A. and Bartol, I. K. (2016). Anti-predator behavior of squid throughout ontogeny. J. Exp. Mar. Biol. Ecol. 480, 26-35.

York, C. A., Bartol, I. K. and Krueger, P. S. (2016). Multiple sensory modalities used by squid in successful predator evasion throughout ontogeny. J. Exp. Biol. 219, 2870-2879. 